\title{
On Resonant Differential Equations with Unbounded Non-Linearities
}

\author{
A. M. Krasnosel'skii, N. A. Kuznetsov and D. I. Rachinskii
}

\begin{abstract}
We present a method to study asymptotically linear degenerate problems with sublinear unbounded non-linearities. The method is based on the uniform convergence to zero of projections of non-linearity increments onto some finite-dimensional spaces. Such convergence was used for the analysis of resonant equations with bounded non-linearities by many authors. The unboundedness of nonlinear terms complicates essentially the analysis of most problems: existence results, approximate methods, systems with parameters, stability, dissipativity, etc. In this paper we present statements on projection convergence for unbounded non-linearities and apply them to various resonant asymptotically linear problems: existence of forced periodic oscillations and unbounded sequences of such oscillations, existence of unbounded solutions, sharp analysis of integral equations with simple degeneration of the linear part (a scalar twopoint boundary value problem is considered as an example), existence of non-trivial cycles for higher order autonomous ordinary differential equations, and Hopf bifurcations at infinity.
\end{abstract}

Keywords: Non-linearity sublinear at infinity, degenerate linear parts, periodic solutions, cycles, integral equations, two-point problems, Hopf bifurcation, existence results

AMS subject classification: 34C25, 45M15, 47H11, 47H30

\section{Projections of non-linearity increments}

1.1 Introduction. Consider the equation $x=T x$ in a Banach space $E$ with an asymptotically linear ${ }^{1)}$ completely continuous operator $T$. If its main linear part $x=A x$ is non-degenerate (has no non-trivial solutions), then various problems related to this equation are rather simple. If 1 is an eigenvalue of the linear operator $A$ (degenerate case), the analysis is much more complicated - it is necessary to use some properties of sublinear non-linearities $F=T-A$. An important property that can be used is the uniform convergence $\Delta F=\phi(F(\xi e+h)-F(\xi e)) \rightarrow 0$ as $\xi \rightarrow \infty$. Here $\phi$ is some linear

All authors: Russian Acad. Sci., Inst. for Inform. Transm. Problems, 19 Bolshoi Karetny lane, Moscow 101447, Russia; amk@iitp.ru, direktor@iitp.ru, rach@iitp.ru

Authors were supported by Grants $\mathrm{N}^{o} \mathrm{~N}^{o}$ 00-01-00571, 01-01-00146, 01-01-06372, and 00-1596116 of the Russian Foundation for Basic Researches. The research was partially done while D. I. Rachinskii was visiting the Regensburg University (Germany), supported by the Research Fellowship from the Alexander von Humboldt Foundation.

1) $T x=A x+F x$ where $A$ is linear and $F$ is sublinear, i.e. the nonlinear operator $F$ satisfies $\|F x\|\|x\|^{-1} \rightarrow 0$ at infinity.

ISSN 0232-2064 / \$2.50 C Heldermann Verlag Berlin 
functional, the convergence must be uniform with respect to all normed eigenvectors $e=A e$ of the operator $A$, and with respect to all vectors $h \in E$ from some special sets depending on $\xi$. The same convergence can be used also in some problems that cannot be reduced directly to equations of the type $x=T x$.

Here we present sufficient conditions for the convergence $\Delta F \rightarrow 0$ for unbounded non-linearities $F$ and consider some applications to ordinary differential equations. Most applications are concerned with periodic and Dirichlet problems for second order equations and for equations arising in control theory. Theorems 1 and 2 on the convergence $\Delta F \rightarrow 0$ are formulated in the space $L^{2}$ for non-linearities $F x(t)=f(t, x(t))$; the uniformity of the convergence is proved for rather special classes of increments $h$. The choice of formulations is determined by the applications. The applications presented are new for the case of unbounded non-linearities. If the non-linearity is bounded, corresponding analogs of our theorems are known.

The paper is organized as follows.

In the next subsection we present three Theorems $1-3$. They are used in all other results of the paper. Especially, in some sense all other results are applications of Theorems 1 and 2. Theorem 3 is slightly more general than Theorems 1 and 2, but we do not present its applications. The last subsection of this section contains some remarks. In Section 2 there are results about existence of forced periodic oscillations. In Section 3 we give a generalization of results by R. Ortega and J. Alonso concerning the existence of unbounded solutions. In Section 4 we consider integral equations and two-point boundary value problems. Sections 5 and 6 are concerned with self-induced oscillations: in Section 5 we present existence theorems for cycles of higher order quasilinear ordinary differential equations while in Section 6 Hopf bifurcations at infinity are considered. In Section 7 we give a lemma to compute some values that we use throughout the paper. The other sections, Sections 8 - 12, contain proofs.

1.2 Convergence conditions. Let $\Theta:[0,+\infty) \rightarrow[1, \infty)$ be a monotone function increasing sublinear at infinity function:

$$
\lim _{\xi \rightarrow+\infty} \frac{\Theta(\xi)}{\xi}=0 .
$$

We suppose that for any constant $M>0$ there exists a number $C=C(M)$ such that $\Theta(M \xi) \leq C \Theta(\xi)$ for all $\xi \geq C$. Let a function $e$ be defined on the interval $[a, b], k>1$ times continuously differentiable. Let its derivative $e^{\prime}$ take the zero value in a finite set of critical points and let the highest order of tangency of the graph of $e$ with a horizontal line equal $k$. The last assumption means that if $e^{\prime}\left(t_{0}\right)=0$, then at least one of the numbers $e^{\prime \prime}\left(t_{0}\right), \ldots, e^{(k)}\left(t_{0}\right)$ is non-zero. Further, let a continuous scalar-valued function $f:[a, b] \times \mathbb{R} \rightarrow \mathbb{R}$ satisfy the estimate

$$
|f(t, x)| \leq c \Theta(|x|) \quad(t \in[a, b], x \in \mathbb{R})
$$

and the Lipschitz condition with respect to the first variable

$$
|f(t, x)-f(s, x)| \leq c \Theta(|x|)|t-s| \quad(t, s \in[a, b], x \in \mathbb{R}) .
$$

At last, let $g:[a, b] \rightarrow \mathbb{R}$ satisfy the Lipschitz condition

$$
|g(t)-g(s)| \leq c|t-s| \quad(s, t \in[a, b])
$$

with some constant $c>0$. 
Theorem 1. Let

$$
\lim _{\xi \rightarrow+\infty} \frac{\Theta^{2 k}(\xi)}{\xi}=0
$$

Then for any $R>0$ the relation

$$
\lim _{\xi \rightarrow \infty} \sup _{\|h\|_{C^{1}} \leq R \Theta(\xi)}\left|\int_{a}^{b} g(t)(f(t, \xi e(t)+h(t))-f(t, \xi e(t))) d t\right|=0
$$

is valid.

Relation (4) is the convergence $\Delta F \rightarrow 0$ for the non-linearity $F x(t)=f(t, x(t))$. With the use of some additional information about the function $f$, it is possible to weaken condition (3). Namely, let $f$ satisfy the Lipschitz condition

$$
|f(t, x)-f(t, y)| \leq d(\xi)|x-y| \quad(|x|,|y| \geq \xi)
$$

where $d(\xi)$ decreases and $d(\xi) \rightarrow 0$ as $\xi \rightarrow \infty$. We suppose that for any $\mu>0$ there exists a number $C_{*}=C_{*}(\mu)$ such that the estimate $d(\mu \xi) \leq C_{*}(\mu) d(\xi)$ holds for all $\xi \geq C_{*}$. We will use this estimate and the relation $\Theta(M \xi) \leq C(M) \Theta(\xi)$ without special references.

Theorem 2. Let $e(\tau) \neq 0$ whenever $e^{\prime}(\tau)=0$, let (1),(2) and (5) be valid and

$$
\lim _{\xi \rightarrow+\infty} \frac{\Theta^{2 k}(\xi) d^{2 k-2}(\xi)}{\xi}=\lim _{\xi \rightarrow+\infty} \frac{\Theta^{2}(\xi)}{\xi}=0 .
$$

Then for any $R>0$ relation (4) holds.

The proofs of Theorems 1 and 2 will be given in Section 8 .

We use the function $\Theta$ in both Theorems 1 and 2 twice: in the estimate for $\|h\|$ in the supremum in (4) and in estimate (1) for the function $f$. In the next theorem we use two different functions in these places.

If relation (4) is valid for some functions $f_{j}$ with the same $\Theta=\Theta(\xi)$, then it is valid for their sum $f^{0}=\sum_{j} f_{j}$. The functions $f_{j}$ may satisfy (1) and (5) with different $\Theta_{j}$ and $d_{j}$; naturally, $f^{0}=\sum_{j} f_{j}$ satisfies (1) with $\Theta=\max _{j} \Theta_{j}$.

Theorem 3. Let $|f(t, x)| \leq c \Theta_{*}(|x|)$ for all $(t, x) \in[a, b] \times \mathbb{R}$ and let either

$$
\lim _{\xi \rightarrow+\infty} \frac{\Theta_{*}^{2 k-1}(\xi) \Theta(\xi)}{\xi}=0,
$$

or $e(\tau) \neq 0$ if $e^{\prime}(\tau)=0$, (5) holds and

$$
\lim _{\xi \rightarrow+\infty} \frac{\Theta_{*}(\xi) \Theta^{2 k-1}(\xi) d^{2 k-2}(\xi)}{\xi}=\lim _{\xi \rightarrow+\infty} \frac{\Theta_{*}(\xi) \Theta(\xi)}{\xi}=0 .
$$

Then for any $R>0$ relation (4) holds. 
In Theorem 3, condition (8) can be more restrictive than condition (7). Applications of this theorem are not considered in the paper. We also do not give the proof; it can be obtained by slight modifications of the proofs of Theorems 1 and 2 .

1.3 Remarks. Here we give the following comments.

A. Lipschitz condition (5) with $d(\xi) \rightarrow 0$ as $\xi \rightarrow+\infty$ is rather restrictive. Under this condition it is possible to prove statements on relation (4), which do not use the derivatives of $e$ and $h$, statements about vector functions [12], etc. Final formulations are weaker than that of Theorem 2 (see [7]). The conclusion of Theorem 2 is valid for functions $e$ satisfying $e(\tau)=e^{\prime}(\tau)=0$ for some $\tau$, the corresponding analog of (6) is more restrictive. In natural applications the assumptions of Theorem 2 are valid. The conclusion of Theorem 2 is also valid for monotone differentiable functions $e$ satisfying $e^{\prime}(t) \geq \eta>0$ in $[a, b]$. In this case, relation (4) follows from $\frac{\Theta^{2}(\xi)}{\xi} \rightarrow 0$ as $\xi \rightarrow+\infty$.

Theorems 1 - 3 can be easily extended for piecewise differentiable functions $e$.

B. The natural case is $k=2$. Theorems 1 - 3 are formulated for arbitrary $k$ due to rare but possible applications. Consider an example. Let $e$ be an eigenfunction of the differential operator $L x=x^{(4)}$ with boundary conditions $x^{\prime}(0)=x^{\prime \prime}(0)=x^{\prime}(1)=$ $x^{\prime \prime}(1)=0$. The leading eigenvalue of this operator is 0 , the corresponding eigenfunction is a constant. Its derivative is identically zero and Theorems $1-3$ are inapplicable. Other eigenvalues (they are equal to $\mu^{4}$, where $\mu$ denotes any non-zero root of the transcendental equation $\cos \mu \cdot \cosh \mu=1$ ) correspond to eigenfunctions $e$ with $k=3$, both Theorems 1 and 2 are applicable.

In general, conditions (6) are different for different $k$. For the function $f=x^{\alpha}+b(t)$ conditions (6) do not depend on $k$ and have the form $2 \alpha<1$.

C. Theorems 1 - 3 can be easily modified if the non-linearity is a sum of functions with different asymptotic properties at infinity. Suppose $f=f_{1}+f_{2}$ and mes $\{t: e(t)=$ $0\}=0$. Let $f_{1}$ satisfy all the conditions of Theorems 1 or 2 and let $f_{2}$ be uniformly bounded. Then for any $R>0$ relation (4) holds.

In the following example Theorem 3 can be used. Let $f_{1}(t, x)=(1+|x|)^{\alpha}$ and $f_{2}(t, x) \leq(1+|x|)^{\beta}$ for some $\beta<\alpha$, let $k=2$ and suppose $f_{2}$ to satisfy condition (5). Then Theorem 1 with $\Theta=(1+\xi)^{\alpha}$ is applicable if $\alpha<\frac{1}{4}$, but Theorem 2 is inapplicable. Theorem 3 with $\Theta=(1+\xi)^{\alpha}$ is applicable if $\alpha<\frac{1}{2}$ and $\beta<\frac{1-\alpha}{3}$, since $f_{1}$ satisfies (1) and (5) with $\Theta_{1}=(1+\xi)^{\alpha}$ and $d_{1}=\alpha(1+\xi)^{\alpha-1}$ and $f_{2}$ satisfies (1) with $\Theta_{2}=(1+\xi)^{\beta}$.

D. Theorems 1 and 2 give sufficient conditions for (4). The necessity of these conditions can be illustrated with the following example. Fix an $\alpha \in(0,1)$ and set

$$
[a, b]=[-1,1], f=(1+|x|)^{\alpha}, \Theta=(1+\xi)^{\alpha}, e=1+t^{2}, h=\xi^{\alpha}, g=1 .
$$

Here $k=2, f^{\prime}=\alpha(1+|x|)^{\alpha-1} \operatorname{sgn} x$ and therefore $d=\alpha(1+\xi)^{\alpha-1}$. The inequality $\alpha<\frac{1}{2}$ is necessary and sufficient for (4) as well as for conditions (6) of Theorem 2 .

E. Let $[a, b]=[0,2 \pi]$ and $e=\sin t$. It would be interesting to obtain sufficient conditions for the relation

$$
\begin{aligned}
\lim _{\xi \rightarrow+\infty} \sup _{\|h\|_{C^{1}} \leq R \Theta(\xi)} \mid \int_{0}^{2 \pi} g(t)(f(t, \xi e(t) & \left.+h(t), \xi e\left(t_{w}\right)+h\left(t_{w}\right)\right) \\
& \left.-f\left(t, \xi e(t), \xi e\left(t_{w}\right)\right)\right) d t \mid=0
\end{aligned}
$$


where $t_{w}=t-w$ if $t \geq w$ and $t_{w}=2 \pi+t-w$ if $t<w$. This relation can be applied to study periodic oscillations in systems with non-linearities $F x(t)=f(t, x(t), x(t-w))$. Some simple conditions are considered in [13].

\section{Existence of forced periodic oscillations}

2.1 Second order equation. Everywhere we use the following

Definition 1. We say that the non-linearity $f$ has a proper growth at infinity if it satisfies (1) and (2), and either relation (3) is valid for $k=2$ or estimate (5) holds and relations (6) are valid for $k=2$.

Consider the equation

$$
x^{\prime \prime}+n^{2} x=f(x)+b(t)
$$

where $n \in \mathbb{N}, b$ is continuous and $2 \pi$-periodic, and $f$ is continuous and unbounded in general. Theorems 4 and 5 below generalize some results from [11] where bounded non-linearities $f$ are considered.

Set

$$
\begin{aligned}
& \Psi(\xi)=\int_{0}^{2 \pi} \sin n t f(\xi \sin n t) d t \\
& \psi_{+}=\liminf _{\xi \rightarrow+\infty}|\Psi(\xi)|, \quad \psi^{+}=\limsup _{\xi \rightarrow+\infty}|\Psi(\xi)| \\
& \bar{b}=\int_{0}^{2 \pi} e^{i n t} b(t) d t .
\end{aligned}
$$

Limits (11) may be finite or infinite, computation of such limits see in Section 7. The function $\Psi$ is always odd.

Theorem 4. Let $f$ in equation (9) have a proper growth at infinity. If the relations

$$
\psi_{+}<|\bar{b}|<\psi^{+}
$$

hold, then equation (9) has an unbounded sequence of $2 \pi$-periodic solutions. If

$$
|\bar{b}|<\psi_{+},
$$

then equation (9) has at least one $2 \pi$-periodic solution and the set of all such solutions is bounded.

The proof of Theorem 4 will be given in Section 9 .

If $\psi^{+}<|\bar{b}|$, then the set of $2 \pi$-periodic solutions of equation (9) is bounded but may be empty. If $|\bar{b}|=\psi_{+}$or $|\bar{b}|=\psi^{+}$, then the knowledge of the values $\psi_{+}, \psi^{+}$and $|\bar{b}|$ is not sufficient to determine whether the set of $2 \pi$-periodic solutions is bounded or not. The special case $|\bar{b}|=\psi_{+}=0$ is essentially different from the case $|\bar{b}|>0$, the corresponding analysis for bounded non-linearities $f$ can be found in [11]. The reasons why these cases are different do not depend on the boundedness or unboundedness of $f$. 
2.2 Control theory equations with delay. Analogs of Theorem 4 are valid for forced oscillations in more complex systems. Let us formulate one statement for control theory equations with delay. For this, consider the equation

$$
L\left(\frac{d}{d t}\right) x(t)=M\left(\frac{d}{d t}\right)\left(f(x(t))+f_{1}(x(t-w))+b(t)\right) .
$$

Here $L$ and $M$ are coprime real polynomials, the degree of $L$ is greater than that of $M$. If $M=1$ and $w=0$, then (14) is a usual higher order ordinary differential equation. Let $L( \pm n i)=0$ for some positive integer $n$ and $L( \pm m i) \neq 0$ for any integer $m \neq n$. Let limits (11) be determined by the function

$$
\Psi(\xi)=\int_{0}^{2 \pi} \sin n t f(\xi \sin n t) d t+\cos n w \int_{0}^{2 \pi} \sin n t f_{1}(\xi \sin n t) d t
$$

Theorem 5. Let both functions $f$ and $f_{1}$ in equation (14) have a proper growth at infinity. If relations (12) are valid, then equation (14) has an unbounded sequence of $2 \pi$-periodic solutions. If relation (13) is valid, then equation (14) has at least one $2 \pi$-periodic solution and the set of all such solutions is bounded.

Theorem 5 generalizes Theorem 4 in two directions: its linear part is more general and there is a delay in the non-linearity. The proofs of both theorems are almost the same, thus we omit that of Theorem 5. Analogs of this theorem for bounded $f$ and $f_{1}$ can be found in [8].

\section{Existence of unbounded solutions}

Consider again equation (9) with $2 \pi$-periodic function $b$. Now we are interested in nonperiodic solutions of (9), namely, we study the existence of unbounded (at $+\infty$ or $-\infty$ ) solutions. This problem was analyzed by J. M. Alonso and R. Ortega for equations with bounded non-linearities [1]. Theorems 1 and 2 allow to obtain similar results for equation (9) with unbounded non-linearities $f$.

Let limits (11) be finite and let

$$
|\bar{b}|>\psi^{+}
$$

For example, limits (11) are finite if the odd part of $f$ is bounded. Let any initial values $x(0)$ and $x^{\prime}(0)$ define a unique solution of equation (9).

Theorem 6. Let $f$ have a proper growth at infinity. Then any solution of equation (9) with sufficiently large $|x(0)|+\left|x^{\prime}(0)\right|$ is unbounded, i.e. at least one of the realtions

$$
\lim _{t \rightarrow \pm \infty}\left(|x(t)|+\left|x^{\prime}(t)\right|\right)=+\infty
$$

holds.

The proof of Theorem 6 will be given in Section 10 .

Theorem 6 is similar to [1: Proposition 3.4]. The main condition (15) is less restrictive than its analog from that Proposition 3.4 even for bounded $f$. From the proof

of Theorem 6 in Section 10 it follows that under its assumptions there exist solutions satisfying both relations (16). 


\section{Integral equations, two-point boundary value problems}

4.1 Integral equation. Consider the integral equation of Hammerstein type

$$
x(t)=\int_{a}^{b} K(t, s) f_{1}(s, x(s)) d s
$$

with non-linearity $f_{1}=\mu x+f(t, x)$, where $\mu \neq 0$ and $f$ is sublinear in $x$. Let the linear operator

$$
(A x)(t)=\int_{a}^{b} K(t, s) x(s) d s
$$

be completely continuous in $L^{2}=L^{2}(a, b)$. If $\mu$ is not a characteristic value of $A$, then equation (17) has at least one solution and the set of all its solutions is bounded.

Let $\mu$ be a simple characteristic value of $A$ (i.e. $\mu^{-1}$ is a simple eigenvalue) and let $e$ be a corresponding normed eigenfunction of $A$. The adjoint operator $A^{*}$ has the eigenfunction $g$ that corresponds to the characteristic value $\mu$, satisfies $(g, e)_{L^{2}}=1$, and is supposed to be Lipschitz continuous. Let $A$ be a continuous operator from $L^{2}$ to $C^{1}$ and from $C^{1}$ to $C^{2}$. Suppose $e^{\prime}(t)=0$ for a finite number of critical points and $e(t) \neq 0$, $e^{\prime \prime}(t) \neq 0$ at these points. Set

$$
\begin{aligned}
& \Psi(\xi)=\int_{0}^{2 \pi} g(t) f(t, \xi e(t)) d t \\
& \varphi_{ \pm}=\liminf _{\xi \rightarrow \pm \infty} \Psi(\xi), \varphi^{ \pm}=\limsup _{\xi \rightarrow \pm \infty} \Psi(\xi) .
\end{aligned}
$$

The following theorem gives sharp conditions for the existence of solutions to equation (17) and for the boundedness of the solution set. Moreover, the index $\operatorname{ind}_{\infty} \Phi$ at infinity $[3,16]$ of the vector field $\Phi(x)=x-\mu A x-A f(\cdot, x)$ in $L^{2}$ is calculated.

Theorem 7. Let the non-linearity $f$ have a proper growth at infinity and let the operator A satisfy all the conditions above. Then:

(i) If either $\varphi^{+}<0<\varphi_{-}$or $\varphi^{-}<0<\varphi_{+}$, then the index at infinity of the vector field $\Phi$ is well defined, $\left|\operatorname{ind}_{\infty} \Phi\right|=1$, and equation (17) has at least one solution.

(ii) If either $\varphi_{-}>0, \varphi_{+}>0$ or $\varphi^{-}<0, \varphi^{+}<0$, then the index at infinity of the vector field $\Phi$ is well defined, ind $\Phi_{\infty} \Phi=0$.

(iii) If either $\varphi_{-}<0<\varphi^{-}$or $\varphi_{+}<0<\varphi^{+}$, or both these relations together are valid, then the index at infinity of the vector field $\Phi$ is not defined and there exists an unbounded sequence of solutions of equation (17) $x_{n} \in L^{2},\left\|x_{n}\right\|_{L^{2}} \rightarrow \infty$.

The proof of Theorem 7 will be given in Section 11 .

The only situation not considered in Theorem 7 is that one of zero limits (18) and neither $\varphi_{-}<0<\varphi^{-}$nor $\varphi_{+}<0<\varphi^{+}$. If $\varphi_{+}<0<\varphi^{+}$, then the unbounded sequence of solutions of equation (17) has the form $x_{n}=\xi_{n} e+h_{n}$ with $\xi_{n} \rightarrow+\infty$ and $\frac{\left\|h_{n}\right\|_{L 2}}{\xi_{n}} \rightarrow 0$ where $\left(g, h_{n}\right)_{L^{2}}=0$. If $\varphi_{-}<0<\varphi^{-}$, then $\xi_{n} \rightarrow-\infty$. If $0 \in\left(\varphi_{-}, \varphi^{-}\right) \cap\left(\varphi_{+}, \varphi^{+}\right)$, then there exist two sequences of solutions $x_{n}^{ \pm}=\xi_{n}^{ \pm} e+h_{n}^{ \pm}$with $\xi_{n}^{ \pm} \rightarrow \pm \infty$. If $\varphi_{-}=\varphi^{-}$and $\varphi_{+}=\varphi^{+}$, then the non-linearity is asymptotically homogeneous in the sense of [6] and the case 3 of Theorem 7 is impossible. 
Some methods to compute limits (18) will be discussed in Section 7 (see also [12]).

4.2 Application to two-point boundary value problems. Consider the problem

$$
\left.\begin{array}{rl}
x^{\prime \prime}+n^{2} x+f(t, x) & =0 \\
x(0)=x(\pi) & =0
\end{array}\right\}
$$

where $n \in \mathbb{N}$. Suppose $f:[0, \pi] \times \mathbb{R} \rightarrow \mathbb{R}$ is continuous in $x \in \mathbb{R}$ and uniformly Lipschitz continuous in $t \in[0, \pi]$. Set

$$
e(t)=\sqrt{\frac{2}{\pi}} \sin n t, \quad \Psi(\xi)=\int_{0}^{\pi} e(t) f(t, \xi e(t)) d t
$$

and define values (18) for this function.

Theorem 8. Let the non-linearity $f$ have a proper growth at infinity. If either $\varphi^{+}<0<\varphi_{-}$or $\varphi^{-}<0<\varphi_{+}$, then problem (19) has at least one solution and the set $K \subset C^{2}$ of all its solutions is bounded. If either $\varphi_{-}<0<\varphi^{-}$or $\varphi_{+}<0<\varphi^{+}$or both these relations together hold, then problem (19) has an infinite number of solutions and the set $K$ of all its solutions is unbounded.

Theorem 8 follows directly from Theorem 7 .

As an illustration, consider the set of solutions of the problem

$$
\left.\begin{array}{rl}
x^{\prime \prime}+n^{2} x+f(t, x)-\lambda e(t) & =0 \\
x(0)=x(\pi) & =0
\end{array}\right\}
$$

with scalar parameter $\lambda$. Define the function $\Psi$ by (20) and let values (18) satisfy

$$
-\infty=\varphi_{-}<\varphi^{-}<0<\varphi_{+}<\varphi^{+}=\infty .
$$

Then for any $\xi \in \mathbb{R}$ there exists at least one $\lambda$ and a function $h,(e, h)_{L^{2}}=0$, such that $x=\xi e+h$ is a solution of problem (21). If $f_{x}^{\prime}$ is sufficiently small, then these $\lambda$ and $h$ are unique for each $\xi$. The set $\left(-\infty, \varphi^{-}\right] \cup\left[\varphi_{+}, \infty\right)$ is that of asymptotic bifurcation points [16] for problem (21). On Figure 1 this set is drawn by thick horizontal lines.

Figure 1: The set of asymptotic bifurcation points for problem (21) 


\section{Cycles in autonomous higher oder ordinary differential equations}

Consider the equation

$$
L\left(\frac{d}{d t}\right) x=f(x)
$$

where $L$ is a real polynomial, $f$ is continuous and sublinear, $f(0)=0$.

Theorem 9. Let the polynomials $L(w i)$ and $\operatorname{Im} L(w i)$ have a pair of common real roots $\pm w_{0} \quad\left(w_{0}>0\right)$ of the same odd multiplicity. Let there exist an interval $\Omega=$ $\left[w_{1}, w_{2}\right] \quad\left(\frac{w_{0}}{2}<w_{1}<w_{0}<w_{2}\right)$ such that

$$
L(n w i) \neq 0 \quad\left(w \in \Omega, n \in \mathbb{N}_{0} \backslash\{1\}\right) \quad \text { and } \quad \operatorname{Im} L(w i) \neq 0 \quad\left(w_{0} \neq w \in \Omega\right),
$$

let

$$
q<\inf _{w \in \Omega} \inf _{n \in \mathbb{N}_{0} \backslash\{1\}}|L(n w i)|
$$

and let the inequality

$$
|\operatorname{Im} L(w i)|\left(|L(n w i)|^{2}-q^{2}\right)+n\left(q^{2}-|\operatorname{Im} L(w i)|^{2}\right) \operatorname{sgn}(\operatorname{Im} L(w i)) \operatorname{Im} L(n w i)>0
$$

be valid for $w=w_{1}, w=w_{2}$ and $2 \leq n \in \mathbb{N}$. Suppose that the non-linearity has a proper growth at infinity, $|f(x)| \leq q|x| \quad(x \in \mathbb{R}), f^{\prime}(0)$ exists with $f^{\prime}(0) \neq 0$ and the function

$$
\Psi(\xi)=\int_{0}^{2 \pi} \sin t f(\xi \sin t) d t
$$

satisfies for all sufficiently large $\xi>0$ the estimate

$$
\Psi(\xi) f^{\prime}(0) \leq-c_{0}<0
$$

Then there exists a non-trivial cycle of equation (9) with period a $T \in\left[\frac{2 \pi}{w_{2}}, \frac{2 \pi}{w_{1}}\right]$.

The proof of Theorem 9 will be given in Section 12 .

Equations $L\left(\frac{d}{d t}\right) x=M\left(\frac{d}{d t}\right) f(x)$ with bounded non-linearities $f$ were considered in [2]. As an example, consider the equation

$$
x^{\prime \prime \prime}+x^{\prime \prime}+x^{\prime}+x=f(x)
$$

where $L(p)=p^{3}+p^{2}+p+1, \operatorname{Im} L(w i)=w\left(1-w^{2}\right)$ and $w_{0}=1$. Suppose that the non-linearity $f$ has a proper growth at infinity, (25) holds for all sufficiently large $\xi$ and $|f(x)| \leq 0.745|x| \quad(x \in \mathbb{R})$. Then equation (26) has at least one cycle with a period $T \in[6.283,7.652]$. 


\section{Hopf bifurcation at infinity}

Consider the differential equation

$$
L\left(\frac{d}{d t}, \lambda\right) x=M\left(\frac{d}{d t}, \lambda\right) f(x, \lambda)
$$

where the real polynomials $L(p, \lambda)$ and $M(p, \lambda)$ of degrees $\ell$ and $m(\ell>m)$ are coprime for each value of the scalar parameter $\lambda \in \Lambda=(a, b)$.

Definition 2. A parameter value $\lambda_{0}$ is called a Hopf bifurcation point at infinity (shortly, a bifurcation point) for equation (27) with a frequency $w_{0}$ if for any sufficiently large $r>0$ there exists a $\lambda_{r}$ such that (27) with $\lambda=\lambda_{r}$ has a $T_{r}$-periodic solution $x_{r}$ and $\lambda_{r} \rightarrow \lambda_{0},\left\|x_{r}\right\|_{C} \rightarrow \infty, T_{r} \rightarrow \frac{2 \pi}{w_{0}}$ as $r \rightarrow \infty$.

The following result is formulated in [9]. Suppose the continuous non-linearity $f(x, \lambda)$ is sublinear in $x$, the polynomial $L$ has a pair of simple conjugate roots $\sigma(\lambda) \pm$ $w(\lambda) i \quad(w(\lambda)>0)$ depending continuously on $\lambda$ where $\sigma\left(\lambda_{0}\right)=0$, and the function $\sigma$ takes values of both sign in every neighborhood of $\lambda_{0}$. Further, suppose $L\left(n w\left(\lambda_{0}\right) i, \lambda_{0}\right) \neq$ 0 for $n \in \mathbb{N}_{0} \backslash\{1\}$. Then $\lambda_{0}$ is a Hopf bifurcation point at infinity for equation (27) with frequency $w\left(\lambda_{0}\right)$.

Here we consider the differential equation

$$
L\left(\frac{d}{d t}\right) x=M\left(\frac{d}{d t}\right) f(x, \lambda)
$$

where the linear part is independent of the parameter. We suppose that the real polynomials $L$ and $M$ are coprime ${ }^{2}$, their degrees satisfy $\ell>m$, and the polynomial $L$ has a pair of imaginary roots $\pm i w_{0} \quad\left(w_{0}>0\right)$. The function $f: \mathbb{R} \times \Lambda \rightarrow \mathbb{R}$ is continuous with respect to its arguments and sublinear uniformly in $\lambda \in \Lambda$, i.e. $|f(x, \lambda)| \leq \Theta(|x|)$ where $\Theta$ is independent of $\lambda$.

Theorem 10. Let the following conditions hold:

1. The number $w_{0}$ is a root of the polynomials $\operatorname{Im}[L(w i) M(-w i)]$ and $L(w i)$ of the same odd multiplicity $K$ and $L\left(n w_{0} i\right) \neq 0$ for every $n \in \mathbb{N}_{0} \backslash\{1\}$.

2. The function $f$ has a proper growth at infinity.

3. For every $\lambda$ there exists the limit

$$
\psi(\lambda)=\lim _{\xi \rightarrow+\infty} \int_{0}^{2 \pi} \sin t f(\xi \sin t, \lambda) d t
$$

and the function $\psi$ takes values of both sign in every neighborhood of the point $\lambda_{0}$.

Then $\lambda_{0}$ is a Hopf bifurcation point at infinity for equation (28) with the frequency $w_{0}$.

We do not give the proof of Theorem 10. A close result is proved in [13] for equations (28) with bounded non-linearities. To obtain the proof for unbounded non-linearities it suffices to combine the method of [13] with Theorems 1 and 2 .

2) Above we supposed this for polynomials depending on a parameter. 
Lemma 1 below explains when limit (29) exists. Assumption 1 of Theorem 10 implies that the polynomial $\operatorname{Im}[L(w i) M(-w i)]$ is non-zero. One can show that the identity $\operatorname{Im}[L(w i) M(-w i)]=0$ holds if and only if both polynomials $L$ and $M$ are even. In this case the equation $L\left(\frac{d}{d t}\right) x=M\left(\frac{d}{d t}\right) f(x)$ with any continuous non-linearity $f$ is Hamiltonian [14, 15]. Moreover, if assumption 1 holds and $f$ is bounded, then this equation has a continuum of periodic cycles $x(\cdot ; \xi)$ such that $\|x(\cdot ; \xi)\|_{C} \rightarrow \infty$ and $T(\xi) \rightarrow \frac{2 \pi}{w_{0}}$ as $\xi \rightarrow \infty$ where $T(\xi)$ is the period of the cycle $x(t ; \xi)$ and $\xi \geq \xi_{0}$ is a parameter. Therefore, the identity $\operatorname{Im}[L(w i) M(-w i)]=0$ implies that all values $\lambda \in \Lambda$ are Hopf bifurcation points at infinity for equation (28) with frequency $w_{0}$. The simplest example of an equation that can be studied with Theorem 10 is $x^{\prime \prime \prime}+x^{\prime \prime}+x^{\prime}+x=f(x, \lambda)$.

\section{Computation of $\varphi_{+}$and $\varphi^{+}$}

In this section, we present algorithms to compute limits (18) for function (19).

Lemma 1. Let $f=f_{1}+f_{2}+f_{3}+f_{4}+f_{5}$ where for the summands the follwing conditons are satisfed:

$f_{1}(x)=\frac{1}{2}[f(x)+f(-x)]$ is the unbounded even part of $f(x)$

$f_{i}(2 \leq i \leq 5)$ are odd and bounded

$f_{2}(x) \rightarrow 0$ as $x \rightarrow \infty$

$f_{3}(x) \rightarrow \pm \bar{f} \neq 0$ as $x \rightarrow \pm \infty$

$f_{4}$ have sublinear primitive $F_{4}: \lim _{x \rightarrow \infty} \frac{F_{4}(x)}{x}=0, F_{4}(x)=\int_{0}^{x} f_{4}(u) d u$.

Then

$$
\begin{aligned}
& \varphi_{+}=4 \bar{f}+\liminf _{\xi \rightarrow+\infty} \int_{0}^{2 \pi} \sin n t f_{5}(\xi \sin n t) d t \\
& \varphi^{+}=4 \bar{f}+\limsup _{\xi \rightarrow+\infty} \int_{0}^{2 \pi} \sin n t f_{5}(\xi \sin n t) d t .
\end{aligned}
$$

Proof. The proof of this simple statement coincides with its analog from [11] for bounded non-linearities. Since $f_{1}$ is even and $f_{2}(x) \rightarrow 0$ as $x \rightarrow \infty$,

$$
\int_{0}^{2 \pi} \sin n t f_{1}(\xi \sin n t) d t=\lim _{\xi \rightarrow \infty} \int_{0}^{2 \pi} \sin n t f_{2}(\xi \sin n t) d t=0 .
$$

The relations $f_{3}(x) \rightarrow \pm \bar{f}$ as $x \rightarrow \pm \infty$ imply

$$
\lim _{\xi \rightarrow+\infty} \int_{0}^{2 \pi} \sin n t f_{3}(\xi \sin n t) d t=4 \bar{f} .
$$

Finally,

$$
\begin{aligned}
\int_{0}^{2 \pi} & \sin n t f_{4}(\xi \sin n t) d t \\
& =4 \int_{0}^{\frac{\pi}{2}} \sin t f_{4}(\xi \sin t) d t \\
& =4 \int_{\varepsilon}^{\frac{\pi}{2}-\varepsilon} \sin t f_{4}(\xi \sin t) d t+4 \int_{[0, \varepsilon] \cup\left[\frac{\pi}{2}-\varepsilon, \frac{\pi}{2}\right]} \sin t f_{4}(\xi \sin t) d t
\end{aligned}
$$




$$
\begin{aligned}
& \leq 4 \xi^{-1} \int_{\varepsilon}^{\frac{\pi}{2}-\varepsilon} \tan t d F_{4}(\xi \sin t)+8 \varepsilon \sup \left|f_{4}(x)\right| \\
& =c \varepsilon+\left.4 \xi^{-1} \tan t F_{4}(\xi \sin t)\right|_{t=\varepsilon} ^{t=\frac{\pi}{2}-\varepsilon}-4 \int_{\varepsilon}^{\frac{\pi}{2}-\varepsilon} \frac{F_{4}(\xi \sin t)}{\xi \cos ^{2} t} d t
\end{aligned}
$$

Since $\varepsilon$ is arbitrarily small and $\frac{F_{4}(x)}{x} \rightarrow 0$ as $x \rightarrow \infty$, this implies

$$
\lim _{\xi \rightarrow+\infty} \int_{0}^{2 \pi} \sin n t f_{4}(\xi \sin n t) d t=0
$$

and the lemma is proved

For example, $f_{4}$ has a sublinear primitive if $f_{4}$ is periodic or almost periodic with zero average; the functions $\sin x^{3}$ and $\sin \sqrt{|x|} \operatorname{sgn} x$ also have sublinear primitives. The primitive of the functions $\sin \ln (1+|x|) \operatorname{sgn} x$ and $\arctan x$ are not sublinear. Nonlinearities with sublinear derivatives were considered in [1] for existence problems of unbounded solutions.

\section{Proof of Theorems 1 and 2}

8.1 Change of variables. The first part of the proof is common for both Theorems 1 and 2. Under their conditions, $\frac{\Theta^{2}(\xi)}{\xi} \rightarrow 0$ as $\xi \rightarrow+\infty$. Consider a decreasing function $\delta: \mathbb{R}^{+} \rightarrow \mathbb{R}^{+}$such that

$$
\lim _{\xi \rightarrow+\infty} \delta(\xi)=0 \quad \text { and } \quad \lim _{\xi \rightarrow+\infty} \frac{\Theta^{2}(\xi)}{\xi \delta^{2 k-2}(\xi)}=0 .
$$

Since $\Theta(\xi) \geq 1$ and $k \geq 2$, this implies also

$$
\lim _{\xi \rightarrow+\infty} \delta_{*}(\xi)=0 \quad \text { where } \quad \delta_{*}(\xi)=\frac{\Theta(\xi)}{\xi \delta^{k}(\xi)} .
$$

Under the assumptions of Theorems 1 and 2 the choice of $\delta$ is different, but it is not essential now.

Let $t_{1}<t_{2}<\ldots<t_{n-1}$ be the set of all critical points of the function $e$ in the interval $(a, b)$, set $t_{0}=a$ and $t_{n}=b$. Consider the intervals

$$
\begin{aligned}
o_{0} & =\left[t_{0}, t_{0}+\delta_{0}\right) \\
o_{j} & =\left(t_{j}-\delta_{j}, t_{j}+\delta_{j}\right) \quad(1 \leq j \leq n-1) \\
o_{n} & =\left(t_{n}-\delta_{n}, t_{n}\right]
\end{aligned}
$$

where $\delta_{j}=\delta(\xi)$ if $e^{\prime}\left(t_{j}\right)=0$ and $\delta_{j}=\delta_{*}(\xi)$ otherwise (the relation $e^{\prime}\left(t_{j}\right) \neq 0$ may hold for $j=0$ and $j=n$ only). The estimate mes $\left\{\cup o_{j}\right\} \leq 2 n \delta+2 \delta_{*}$ implies mes $\left\{\cup o_{j}\right\} \rightarrow 0$ as $\xi \rightarrow+\infty$. Suppose that $\xi$ is so large that the closures of the intervals $o_{j}$ are disjoint. Then the set $[a, b] \backslash \cup o_{j}$ is the unification of $n$ disjoint segments $\left[a_{j}, b_{j}\right] \subset\left(t_{j}, t_{j+1}\right)$ (the points $a_{j}$ and $b_{j}$ depend on $\xi$, the segments $\left[a_{j}, b_{j}\right]$ become larger as $\xi$ increases). 
By construction, $\left[a_{j}-\frac{\delta_{j}}{2}, b_{j}+\frac{\delta_{j}}{2}\right] \subset\left(t_{j}, t_{j+1}\right)$ for each $j$ and the function $e$ is strictly monotone on every interval $\left(t_{j}, t_{j+1}\right)$. Moreover, the relations $e^{(\ell)}\left(t_{j}\right) \neq 0$ with $\ell=\ell_{j} \leq$ $k$ imply the estimate

$$
\left|e^{\prime}(t)\right| \geq c_{1} \delta^{k-1} \quad\left(t \in\left[a_{j}-\frac{\delta_{j}}{2}, b_{j}+\frac{\delta_{j}}{2}\right] ; j=0, \ldots, n-1\right)
$$

where $c_{1}>0$ is independent of $\xi$.

To prove relation (4) we estimate separately the integrals over the sets $\cup\left[a_{j}, b_{j}\right]$ and $\cup o_{j}$ and show that for some appropriate choice of $\delta$ both integrals tend to zero as $\xi \rightarrow+\infty$. First, fix some $j$, consider the segment $\left[a_{j}, b_{j}\right]$ and the corresponding integral

$$
I\left(a_{j}, b_{j}\right)=\int_{a_{j}}^{b_{j}} g(t)(f(t, \xi e(t)+h(t))-f(t, \xi e(t))) d t .
$$

Since the function $e$ is strictly monotone on the segment $\left[a_{j}-\frac{\delta_{j}}{2}, b_{j}+\frac{\delta_{j}}{2}\right]$, the inverse function $e^{-1}$ is well defined on the segment $e\left(\left[a_{j}-\frac{\delta_{j}}{2}, b_{j}+\frac{\delta_{j}}{2}\right]\right)$ and (32) implies

$$
\left|\frac{d}{d \tau} e^{-1}(\tau)\right| \leq c_{1}^{-1} \delta^{1-k} \quad\left(\tau \in e\left(\left[a_{j}-\frac{\delta_{j}}{2}, b_{j}+\frac{\delta_{j}}{2}\right]\right)\right) .
$$

From $(31)$ and $\|h\|_{C^{1}} \leq R \Theta(\xi)$ the relations

$$
\xi^{-1}\|h\|_{C^{1}}=\left\{\begin{array}{l}
o\left(\delta^{k}\right) \\
o\left(\delta^{k-1} \delta_{*}\right)
\end{array} \quad(\xi \rightarrow+\infty)\right.
$$

follow, therefore estimate (32) implies that the function $e+\xi^{-1} h$ is strictly monotone on the segment $\left[a_{j}, b_{j}\right]$, their values belong to the segment $e\left(\left[a_{j}-\frac{\delta_{j}}{2}, b_{j}+\frac{\delta_{j}}{2}\right]\right)$ and

$$
\left|e^{\prime}(t)+\xi^{-1} h^{\prime}(t)\right| \geq c_{1} \frac{\delta^{k-1}}{2} \quad\left(t \in\left[a_{j}, b_{j}\right]\right)
$$

if $\xi$ is sufficiently large. Let us change the variable in integral (33) by the formula $e(\tau)=e(t)+\xi^{-1} h(t)$; the change $t \rightarrow \tau$ is correct due to the monotonicity of both sides of this formula.

Let us prove two preliminary estimates. Namely, the relations

$$
\tau=e^{-1}\left(e(t)+\xi^{-1} h(t)\right)=t+e^{-1}\left(e(t)+\xi^{-1} h(t)\right)-e^{-1}(e(t))
$$

and (34) imply the estimate $|\tau-t| \leq c_{1}^{-1} \xi^{-1} \delta^{1-k}|h(t)|$, therefore $|\tau-t| \leq r_{1} \frac{\Theta(\xi)}{\xi \delta^{k-1}(\xi)}$ (here and everywhere $r_{m}>0$ are constants the exact values of which do not play any role). The next preliminary estimate follows from the formula $e^{\prime}(\tau)=\left(e^{\prime}(t)+\xi^{-1} h^{\prime}(t)\right) t^{\prime}(\tau)$ which implies

$$
\begin{aligned}
\left|t^{\prime}(\tau)-1\right| & =\left|\frac{e^{\prime}(\tau)}{e^{\prime}(t)+\xi^{-1} h^{\prime}(t)}-1\right| \\
& =\left|\frac{e^{\prime}(\tau)-e^{\prime}(t)-\xi^{-1} h^{\prime}(t)}{e^{\prime}(t)+\xi^{-1} h^{\prime}(t)}\right| \\
& \leq r_{2} \frac{|\tau-t|+\frac{\Theta(\xi)}{\xi}}{\delta^{k-1}(\xi)} \\
& \leq r_{3} \frac{\Theta(\xi)}{\xi \delta^{2 k-2}(\xi)} .
\end{aligned}
$$


From (30) we see that $t^{\prime}(\tau)$ is close to 1 for large $\xi$.

Now let us come back to integral (33). Since

$$
\int_{a_{j}}^{b_{j}} g(t) f(t, \xi e(t)+h(t)) d t=\int_{\tau\left(a_{j}\right)}^{\tau\left(b_{j}\right)} g(t(\tau)) f(t(\tau), \xi e(\tau)) t^{\prime}(\tau) d \tau
$$

it follows that $I\left(a_{j}, b_{j}\right) \leq \varepsilon_{1}+\varepsilon_{2}+e_{3}+\varepsilon_{4}$ where

$$
\begin{aligned}
& \varepsilon_{1}=\left|\int_{\tau\left(a_{j}\right)}^{\tau\left(b_{j}\right)} g(t(\tau)) f(t(\tau), \xi e(\tau)) t^{\prime}(\tau) d \tau-\int_{a_{j}}^{b_{j}} g(t(\tau)) f(t(\tau), \xi e(\tau)) t^{\prime}(\tau) d \tau\right| \\
& \varepsilon_{2}=\left|\int_{a_{j}}^{b_{j}} g(t(\tau)) f(t(\tau), \xi e(\tau)) t^{\prime}(\tau) d \tau-\int_{a_{j}}^{b_{j}} g(\tau) f(t(\tau), \xi e(\tau)) t^{\prime}(\tau) d \tau\right| \\
& \varepsilon_{3}=\left|\int_{a_{j}}^{b_{j}} g(\tau) f(t(\tau), \xi e(\tau)) t^{\prime}(\tau) d \tau-\int_{a_{j}}^{b_{j}} g(\tau) f(\tau, \xi e(\tau)) t^{\prime}(\tau) d \tau\right| \\
& \varepsilon_{4}=\left|\int_{a_{j}}^{b_{j}} g(\tau) f(\tau, \xi e(\tau)) t^{\prime}(\tau) d \tau-\int_{a_{j}}^{b_{j}} g(\tau) f(\tau, \xi e(\tau)) d \tau\right| .
\end{aligned}
$$

Estimates (1) - (2) and $|g(t)-g(s)| \leq|t-s|$ imply

$$
\varepsilon_{1}, \varepsilon_{2}, \varepsilon_{3} \leq r_{4} \Theta(\xi) \max |\tau-t(\tau)| \quad \text { and } \quad \varepsilon_{4} \leq r_{5} \Theta(\xi) \max \left|t^{\prime}(\tau)-1\right|
$$

and from the above estimates for $|\tau-t(\tau)|$ and $\left|t^{\prime}(\tau)-1\right|$ we conclude

$$
\varepsilon_{1}, \varepsilon_{2}, \varepsilon_{3} \leq r_{6} \frac{\Theta^{2}(\xi)}{\xi \delta^{k-1}(\xi)}, \quad \varepsilon_{4} \leq r_{7} \frac{\Theta^{2}(\xi)}{\xi \delta^{2 k-2}(\xi)}, \quad I\left(a_{j}, b_{j}\right) \leq r_{8} \frac{\Theta^{2}(\xi)}{\xi \delta^{2 k-2}(\xi)} .
$$

Therefore relation (30) implies that all integrals (33) vanish as $\xi \rightarrow+\infty$.

8.2 Completion of the proof. It remains to show that the integral

$$
J(\xi)=\int_{\cup o_{j}} g(t)(f(t, \xi e(t)+h(t))-f(t, \xi e(t))) d t
$$

vanishes as $\xi \rightarrow+\infty$ uniformly with respect to $h,\|h\|_{C^{1}} \leq R \Theta(\xi)$, for some appropriate $\delta(\xi)$ satisfying (30). Under the conditions of Theorem 1 set

$$
\delta(\xi)=\frac{1}{\Theta(\xi)}\left(\frac{\Theta^{2 k}(\xi)}{\xi}\right)^{\frac{1}{3 k-3}}
$$

Then $\frac{\Theta^{2}(\xi)}{\xi \delta^{2 k-2}(\xi)}=\left(\frac{\Theta^{2 k}(\xi)}{\xi}\right)^{\frac{1}{3}}$. Due to (3), these relations and the estimates

$$
|J(\xi)| \leq r_{9} \Theta(\xi) \operatorname{mes}\left\{\cup o_{j}\right\} \leq r_{9} \Theta(\xi)\left(2 n \delta(\xi)+2 \delta_{*}(\xi)\right) \leq r_{10}\left(\Theta(\xi) \delta(\xi)+\frac{\Theta^{2}(\xi)}{\xi \delta^{2 k-2}(\xi)}\right)
$$


imply (30) and $J(\xi) \rightarrow 0$ as $\xi \rightarrow+\infty$. Theorem 1 is proved.

Suppose that the conditions of Theorem 2 are satisfied. Consider an interval $o_{j}$. If $e^{\prime}\left(t_{j}\right)=0$, then by assumption of this theorem $e\left(t_{j}\right) \neq 0$ and relation (5) implies for $t \in o_{j}$

$$
\begin{aligned}
|f(t, \xi e(t)+h(t))-f(t, \xi e(t))| & \leq d\left(\min _{t \in \cup o_{j}}\{|\xi e(t)|,|\xi e(t)+h(t)|\}\right)|h(t)| \\
& \leq r_{11} d(\xi) \Theta(\xi),
\end{aligned}
$$

hence

$$
\begin{aligned}
\left|\int_{o_{j}} g(t)(f(t, \xi e(t)+h(t))-f(t, \xi e(t))) d t\right| & \leq r_{12} d(\xi) \Theta(\xi) \delta_{j}(\xi) \\
& =r_{12} d(\xi) \Theta(\xi) \delta(\xi) .
\end{aligned}
$$

The relation $e^{\prime}\left(t_{j}\right) \neq 0$ may be valid for $j=0$ and $j=n$; in these cases $\delta_{j}=\delta_{*}(\xi)$ and

$$
\left|\int_{o_{j}} g(t)(f(t, \xi e(t)+h(t))-f(t, \xi e(t))) d t\right| \leq r_{13} \Theta(\xi) \delta_{*}(\xi) \leq r_{13} \frac{\Theta^{2}(\xi)}{\xi \delta^{2 k-2}(\xi)} .
$$

Therefore

$$
|J(\xi)| \leq r_{14}\left(d(\xi) \Theta(\xi) \delta(\xi)+\frac{\Theta^{2}(\xi)}{\xi \delta^{2 k-2}(\xi)}\right) .
$$

Under the conditions of Theorem 2 we set

$$
\delta(\xi)=\left(\frac{\Theta^{2}(\xi)}{\xi}\right)^{\frac{1}{3 k-3}} \min \left\{(d(\xi) \Theta(\xi))^{-\frac{1}{3}}, 1\right\} .
$$

Then

$$
\frac{\Theta^{2}(\xi)}{\xi \delta^{2 k-2}(\xi)}=\max \left\{\left(\frac{\Theta^{2 k}(\xi) d^{2 k-2}(\xi)}{\xi}\right)^{\frac{1}{3}},\left(\frac{\Theta^{2}(\xi)}{\xi}\right)^{\frac{1}{3}}\right\}
$$

and

$$
d(\xi) \Theta(\xi) \delta(\xi)=\left\{\begin{array}{ll}
\left(\frac{\Theta^{2 k}(\xi) d^{2 k-2}(\xi)}{\xi}\right)^{\frac{1}{3 k-3}} & \text { if } d(\xi) \Theta(\xi) \geq 1 \\
\Theta(\xi)\left(\frac{\Theta^{2}(\xi)}{\xi}\right)^{\frac{1}{3 k-3}} & \text { if } d(\xi) \Theta(\xi)<1
\end{array},\right.
$$

i.e.

$$
d(\xi) \Theta(\xi) \delta(\xi) \leq\left(\frac{\Theta^{2}(\xi)}{\xi \delta^{2 k-2}(\xi)}\right)^{\frac{1}{k-1}} .
$$

Therefore relations (6) imply (30) and $J(\xi) \rightarrow 0$ as $\xi \rightarrow+\infty$. Theorem 2 is also proved. 


\section{Proof of Theorem 4}

9.1 General scheme. The proof consists of several steps. First we formulate a simple auxiliary statement on the explicit form of the superposition operator in some 2dimensional subspace of $L^{2}$. Then the periodic problem is replaced by the equivalent operator equation

$$
x=A(\mu x-f(x)-b)
$$

of Hammerstein type with a proper linear operator $A$ and a proper $\mu$.

In the next step, the index at infinity of the vector field $x-A(\mu x-f(x)-b)$ is calculated for the case $\psi_{+}>|\bar{b}|$. It turns out that the index is not zero which proves the second part of Theorem 4 . The index is calculated by the usual homotopic methods: all the zeroes of some deformation $\Phi=\Phi(\lambda, x)$ satisfy an a priori estimate, $\Phi(1, x)=x-A(\mu x-f(x)-b)$, and $\Phi(0, \cdot)$ is a vector field of Landesman-Lazer type. The index of such vector fields was calculated, e.g., in $[5,17,18]$.

The case $\psi_{+}<|\bar{b}|<\psi^{+}$is more cumbersome. We prove that for any $\xi_{0}$ there exist $\xi_{*}>\xi_{0}$ and $\xi^{*}>\xi_{*}$ such that equation (36) has at least one solution $x(t)=$ $\xi \sin (n t+\lambda)+h(t)$ with $\xi \in\left(\xi_{+}, \xi^{*}\right)$. This proves the first part of Theorem 4 .

9.2 Planar mapping. If $x$ is a $2 \pi$-periodic solution of equation (9), then

$$
\int_{0}^{2 \pi}(f(x(t))+b(t)) \cos n t d t=\int_{0}^{2 \pi}(f(x(t))+b(t)) \sin n t d t=0 .
$$

Consider the orthogonal projector $P$ defined by

$$
(P u)(t)=\frac{1}{\pi} \int_{0}^{2 \pi} \cos n(t-\tau) u(\tau) d \tau
$$

in $L^{2}=L^{2}(0,2 \pi)$. Equalities (37) are equivalent to $P f(x)+P b=0$. Denote by $\Pi_{n}$ the 2-dimensional subspace of $L^{2}$ spanned on the functions $\cos n t$ and $\sin n t$. By definition, $P L^{2}=\Pi_{n}$ and any $e \in \Pi_{n}$ has the form $e=\xi \sin (n t+\lambda)$ where $\xi \geq 0$ and $\lambda \in[0,2 \pi)$. The proof of the following lemma is by simple computations and we omit it.

Lemma 2. For every $\xi \geq 0$ and $\theta \in[0,2 \pi)$,

$$
P f(\xi \sin (n t+\theta))=\frac{1}{\pi} \Psi(\xi) \sin (n t+\theta)
$$

where $\Psi$ is defined by $(10)$.

9.3 Equivalent integral equation. Consider the linear operator $x=A u$ that maps any function $u \in L^{2}$ to a unique solution $x$ of the problem

$$
\left.\begin{array}{c}
-x^{\prime \prime}+x=u \\
x(0)=x(2 \pi), x^{\prime}(0)=x^{\prime}(2 \pi)
\end{array}\right\} .
$$

This operator acts in $L^{2}$, it is completely continuous in $L^{2}$, self-adjoint, and positive definite. Moreover, $A$ is an integral operator which can be considered in various functional 
spaces: it is continuous as an operator from $L^{2}(0,2 \pi)$ to $C^{1}(0,2 \pi)$ and from $C(0,2 \pi)$ to $C^{2}(0,2 \pi)$. Its spectrum is the sequence of eigenvalues $n^{2}+1 \quad\left(n \in \mathbb{N}_{0}\right)$. The eigenvalue 1 is simple, it corresponds to constant eigenfunctions; the other eigenvalues have the multiplicity 2. The eigenvalue $n^{2}+1$ corresponds to the eigenfunctions $\xi \sin (n t+\theta)$ with arbitrary $\xi>0$ and $\theta \in[0,2 \pi)$, these eigenfunctions and the zero function form the plane $\Pi_{n}$ in $L^{2}$.

Now we can rewrite the $2 \pi$-periodic problem for equation (9) equivalently in form (36) with $\mu=n^{2}+1$. A function $x \in L^{2}$ is a solution of operator equation (36) if and only if it is a classical $2 \pi$-periodic solution of equation (9).

The principal linear part $x=\mu A x$ of equation (36) is degenerate at infinity: the linear operator $I-\mu A$ has the non-trivial kernel $\Pi_{n}$. Recall that $P$ is the orthogonal projector onto the plane $\Pi_{n}$ in $L^{2}$. Set $Q=I-P$.

9.4 The case $\varphi_{+}>|\bar{b}|$. This estimate implies $\Psi(\xi) \neq 0$ and has the same sign for all sufficiently large $\xi$. Fix an $a \in \mathbb{R}$ such that $\psi_{+}>|a|>|\bar{b}|$ and $a \Psi(\xi)>0$ for large $\xi$. Define the function

$$
s(\xi)= \begin{cases}1 & \text { if } \xi \geq 1 \\ \xi & \text { if }|\xi|<1 \\ -1 & \text { if } \xi \leq-1\end{cases}
$$

and consider the deformation

$$
\Phi(\lambda, x)=x-A\left(\mu x-\lambda f(x)-\frac{a(1-\lambda) s(x)}{4}-b\right) .
$$

To prove the existence of at least one solution of equation (36), it suffices to establish the two facts:

- to prove that all the zeroes of the deformation $\Phi$ satisfy an a priori estimate

- to show that the index at infinity of the vector field $\Phi(0, x)$ is non-zero.

We begin with the a priori estimate. Let $x(t)=\xi \sin (n t+\theta)+h(t)$ where $h=Q x$, and let $\Phi(\lambda, x)=0$. Then $Q \Phi(\lambda, x)=0$ and $P \Phi(\lambda, x)=0$. Since the linear operator $I-\mu A$ is continuously invertible in the subspace $Q L^{2} \subset L^{2}$, the equality $Q \Phi(\lambda, x)=0$ implies the estimate $\|h\|_{C^{1}} \leq c \Theta(\xi)$. The equality $P \Phi(\lambda, x)=0$ can be rewritten as

$$
\lambda P f(x)+\frac{(1-\lambda) a P s(x)}{4}=-P b .
$$

Theorems 1 and 2 imply $P f(x)-P f(\xi \sin (n t+\theta)) \rightarrow 0$ and similarly $P s(x)-P s(\xi \sin (n t+\square$ $\theta)) \rightarrow 0$ as $\xi \rightarrow+\infty$. From (38)

$$
\begin{aligned}
& P f(\xi \sin (n t+\theta))=\frac{1}{\pi} \Psi(\xi) \sin (n t+\theta) \\
& P s(\xi \sin (n t+\theta))=\left(\frac{4}{\pi}+O\left(\xi^{-1}\right)\right) \sin (n t+\theta)
\end{aligned}
$$

follows. Therefore

$$
\liminf _{\xi \rightarrow+\infty}\left\|\lambda P f(x)+\frac{(1-\lambda) a P s(x)}{4}\right\|_{L^{2}}=\liminf _{\xi \rightarrow+\infty} \frac{1}{\sqrt{\pi}}|\lambda \Psi(\xi)+(1-\lambda) a| \geq \frac{|a|}{\sqrt{\pi}} .
$$


Since $\sqrt{\pi}\|P b\|_{L^{2}}=|\bar{b}|<|a|$, relation (40) is not true for large $\xi$. This proves the required a priori estimate.

Now consider the vector field $\Phi(0, x)=x-A\left(\mu x-\frac{a s(x)}{4}-b\right)$ where the non-linearity satisfies $s(\xi) \rightarrow \pm 1$ as $\xi \rightarrow \pm \infty$. The computation of the index at infinity of such vector fields can be found, for example, in $[5,17,18]$. It is proved there that $|\operatorname{ind} \Phi(0, \cdot)|=1$ if $|a|>|\bar{b}|$.

The a priori estimate guarantees that ind $\Phi(\lambda, \cdot)$ does not depend on $\lambda$. Therefore,

$$
\text { ind } \Phi(1, \cdot)=\text { ind } \Phi(0, \cdot) \neq 0
$$

and the conclusion of Theorem 4 follows from the general degree theory.

9.5 Unbounded sequence of solutions. Suppose that estimates (12) holds. Then there exist unbounded sequences $\left\{\xi_{k}\right\}$ and $\left\{\xi^{k}\right\}$ such that $\xi_{k}<\xi^{k}<\xi_{k+1}$ and

$$
\left|\Psi\left(\xi_{k}\right)\right|+\varepsilon<|\bar{b}|<\left|\Psi\left(\xi^{k}\right)\right|-\varepsilon
$$

for some $\varepsilon>0$. Without loss of generality, suppose that all $\xi_{k}$ are sufficiently large and therefore the supremum in (4) for $[a, b]=[0,2 \pi]$ as well as the trigonometric functions $e$ and $g$ are sufficiently small for all $\xi \geq \xi_{k}$.

Let us show that the field $x-A(\mu x-f(x)-b)$ is non-zero on the boundary $\partial \Omega_{k}$ of the set $\Omega_{k} \subset L^{2}$,

$$
\Omega_{k}=\left\{x=\xi \sin (n t+\theta)+h(t): h=Q x,\|h\|_{L^{2}} \leq R_{1} \Theta\left(\xi^{k}\right)+1, \xi_{k} \leq \xi \leq \xi^{k}\right\}
$$

with a proper $R_{1}>0$, and that the rotation $\gamma_{k}$ of this vector field on $\partial \Omega_{k}$ is either 1 or -1 . The relation $\gamma_{k} \neq 0$ implies that each set $\Omega_{k}$ contains at least one solution of equation (36); since the sets $\Omega_{k}$ are disjoint for different $k$ and $\xi_{k} \rightarrow+\infty$, this implies the conclusion of Theorem 4 .

Let us calculate the value $\left|\gamma_{k}\right|$ for some fixed $k$. Consider the deformation

$$
\Xi(\lambda, x)=x-\mu A x+A f(x)+A b+\lambda A(P f(P x)-f(x)) \quad(\lambda \in[0,1]) .
$$

For $\lambda=0$ and $\lambda=1$,

$$
\begin{aligned}
& \Xi(0, x)=x-A(\mu x-f(x)-b) \\
& \Xi(1, x)=x-A(\mu x-P f(P x)-b) .
\end{aligned}
$$

First we prove that the deformation $\Xi$ is non-zero on $\partial \Omega_{k}$. Let $\Xi(\lambda, x)=0$ for some $\lambda \in[0,1]$ and $x=\xi \sin (n t+\theta)+h(t)$ with $h=Q x$. Then $Q \Xi(\lambda, x)=0$ and $P \Xi(\lambda, x)=0$. The first equality implies the estimates

$$
\|h\|_{L^{2}} \leq R_{1} \Theta(\xi) \quad \text { and } \quad\|h\|_{C^{1}} \leq c \Theta(\xi)
$$

where the constants $c>0$ and $R_{1}>0$ are independent of $\lambda, \xi, k$. We use this $R_{1}$ in the definition of $\Omega_{k}$. Therefore the relations $\|h\|_{L^{2}}=R_{1} \Theta\left(\xi^{k}\right)+1 \quad\left(\xi_{k} \leq \xi \leq \xi^{k}\right)$ imply $Q \Xi(\lambda, x) \neq 0$. 
The rest of the boundary $\partial \Omega_{k}$ consists of functions $x=\xi \sin (n t+\theta)+h(t)$ with $\xi=\xi_{k}$ and $\xi=\xi^{k}$. The equality $P \Xi(\lambda, x)=0$ can be rewritten as

$$
P[f(P x)+b]=(1-\lambda) P[f(P x)-f(x)] .
$$

By Lemma 2, its left-hand side is

$$
P[f(P x)+b]=\frac{1}{\pi} \Psi(\xi) \sin (n t+\theta)+P b
$$

and due to (41) its norm satisfies $\sqrt{\pi}\|P[f(P x)+b]\|_{L^{2}}>\varepsilon$ for $\xi=\xi_{k}$ and $\xi=\xi^{k}$. The right-hand side $(1-\lambda) P[f(P x)-f(x)]$ vanishes as $\xi \rightarrow+\infty$ according to Theorems 1 and 2, therefore the relation $\Xi(\lambda, x)=0$ is impossible for sufficiently large $\xi=\xi_{k}$ and $\xi=\xi^{k}$.

Thus, $\Xi(\lambda, x) \neq 0$ on $\partial \Omega_{k}$. Therefore the rotation $\gamma\left(\Xi(\lambda, \cdot), \partial \Omega_{k}\right)$ of the vector field $\Xi(\lambda, \cdot)$ on $\partial \Omega_{k}$ is the same for each $\lambda$. For $\lambda=1$ this rotation can be computed by the rotation product formula (see [16: p. 117/Theorem 22.4] or [3: p. 72/ Theorem 3.15]) which in our case has the form

$$
\gamma\left(\Xi(1, \cdot), \partial \Omega_{k}\right)=(-1)^{\alpha} \gamma\left(P f(\cdot)+P b, \partial Z_{k}\right) .
$$

Here $\alpha$ is an integer and $\gamma\left(P f(\cdot)+P b, \partial Z_{k}\right)$ is the rotation of the vector field $P f(x)+P b$ on the boundary $\partial Z_{k}$ of the annulus

$$
Z_{k}=\left\{x=\xi \sin (n t+\theta): \xi_{k} \sqrt{\pi} \leq\|x\|_{L^{2}}=\xi \sqrt{\pi} \leq \xi^{k} \sqrt{\pi}\right\} \subset \Pi_{n} .
$$

This rotation is defined by the formula $\gamma\left(P f(\cdot)+P b, \partial Z_{k}\right)=\gamma_{2}-\gamma_{1}$, where $\gamma_{1}$ and $\gamma_{2}$ are the rotations of the field $P f(x)+P b$ on the circles $\|x\|_{L^{2}}=\xi_{k} \sqrt{\pi}$ and $\|x\|_{L^{2}}=$ $\xi^{k} \sqrt{\pi}\left(x \in \Pi_{n}\right)$. By Lemma 2,

$$
P f(x)=\frac{\Psi(\xi)}{\pi \xi} x \quad \text { and } \quad\|x\|_{L^{2}}=\xi \sqrt{\pi} \quad\left(x \in \Pi_{n}\right),
$$

hence relations $\sqrt{\pi}\|P b\|_{L^{2}}=\bar{b}$ and (41) imply

$$
\begin{aligned}
& \|P f(x)\|_{L^{2}}<\|P b\|_{L^{2}},\|x\|_{L^{2}}=\xi_{k} \sqrt{\pi} \\
& \|P f(x)\|_{L^{2}}>\|P b\|_{L^{2}},\|x\|_{L^{2}}=\xi^{k} \sqrt{\pi} .
\end{aligned}
$$

By the Rouché theorem [16] it follows from these estimates that $\gamma_{1}$ is equal to the rotation of the constant vector field $P b$ on the circle $\|x\|_{L^{2}}=\xi_{k} \sqrt{\pi}$, i.e. $\gamma_{1}=0$. The rotation $\gamma_{2}$ is equal to the rotation of field (43) on the circle $\|x\|_{L^{2}}=\xi^{k} \sqrt{\pi}$, i.e. $\gamma_{2}=1$. Therefore,

$$
\gamma_{k}=\gamma\left(\Xi(1, \cdot), \partial \Omega_{k}\right)=(-1)^{\alpha}\left(\gamma_{2}-\gamma_{1}\right)=(-1)^{\alpha} .
$$

Theorem 4 is completely proved. 


\section{Proof of Theorem 6}

Denote by $x(t ; \zeta, \eta)$ the solution of equation (9) satisfying $x(0 ; \zeta, \eta)=\zeta$ and $x^{\prime}(0 ; \zeta, \eta)$ $=\eta$. Denote by $\mathcal{P}: \mathbb{R}^{2} \rightarrow \mathbb{R}^{2}$ the operator of translation along the trajectories of equation (9) from the moment 0 to the moment $2 \pi$. This operator maps any initial point $\{\zeta, \eta\}$ to the point $\left\{x(2 \pi), x^{\prime}(2 \pi)\right\}$ of the trajectory of the solution $x(t)=x(t ; \zeta, \eta)$ in the phase plane $\mathbb{R}^{2}$.

Consider the function

$$
h(t)=h(t ; \zeta, \eta):=x(t ; \zeta, \eta)-\zeta \cos n t-\frac{\eta}{n} \sin n t
$$

This function is the solution of the Cauchy problem

$$
\left.\begin{array}{rl}
h^{\prime \prime}+n^{2} h & =f(x(t ; \zeta, \eta))+b(t) \\
(0)=h^{\prime}(0) & =0
\end{array}\right\}
$$

hence it satisfies

$$
\begin{aligned}
h(t) & =\frac{1}{n} \int_{0}^{t} \sin n(t-\tau)(f(x(\tau ; \zeta, \eta))+b(\tau)) d \tau \\
h^{\prime}(t) & =\int_{0}^{t} \cos n(t-\tau)(f(x(\tau ; \zeta, \eta))+b(\tau)) d \tau
\end{aligned}
$$

and therefore

$$
\|h\|_{C^{1}} \leq R_{0} \Theta(|\zeta|+|\eta|)
$$

where $R_{0}>0$ is independent of $\zeta$ and $\eta$. By Theorems 1 and 2,

$$
\lim _{\xi \rightarrow \infty\|h\|_{C^{1}} \leq R \Theta(\xi)} \sup _{\xi}\left|\int_{0}^{2 \pi} \sin (n t+\theta) f\left(\xi \sin \left(n t+\theta_{1}\right)+h(t)\right) d t-\Psi(\xi) \cos \left(\theta_{1}-\theta\right)\right|=0
$$

and the convergence herein is uniform with respect to all $\theta$ and $\theta_{1}$, for each $R>0$. Relations (44) - (46) imply for each $\varepsilon>0$ the existence of a $\xi_{0}=\xi_{0}(\varepsilon)$ such that the inequality

$$
\int_{0}^{2 \pi} \sin (n t+\theta) f(x(t ; \zeta, \eta)) d t \leq \psi^{+}+\varepsilon
$$

is valid for all $\theta \in[0,2 \pi)$ whenever $|\zeta|+|\eta| \geq \xi_{0}$.

Set $\varepsilon=\frac{|\bar{b}|-\psi^{+}}{3}$. Estimate (15) implies $\varepsilon>0$. Let $\theta$ be any number such that

$$
-\int_{0}^{2 \pi} b(t) \sin (n t+\theta) d t \geq|\bar{b}|-\varepsilon=\psi^{+}+2 \varepsilon
$$

Consider the linear guiding function

$$
V(\zeta, \eta ; \theta)=-\eta \sin \theta+n \zeta \cos \theta
$$


on the plane $\{\zeta, \eta\} \in \mathbb{R}^{2}$. If $V(\zeta, \eta ; \theta)>0$ is sufficiently large, then $|\zeta|+|\eta| \geq \xi_{0}(\varepsilon)$ and therefore

$$
\begin{aligned}
V(\mathcal{P}(\zeta, \eta) ; \theta)-V(\zeta, \eta ; \theta) \\
\quad=V\left(x(2 \pi ; \zeta, \eta), x^{\prime}(2 \pi ; \zeta, \eta) ; \theta\right)-V(\zeta, \eta ; \theta) \\
\quad=n h(2 \pi ; \zeta, \eta) \cos \theta-h^{\prime}(2 \pi ; \zeta, \eta) \sin \theta \\
\quad=-\int_{0}^{2 \pi} \sin (n t+\theta) b(t) d t-\int_{0}^{2 \pi} \sin (n t+\theta) f(x(t ; \zeta, \eta)) d t \\
\quad \geq \varepsilon
\end{aligned}
$$

Similarly, if $-V(\zeta, \eta ; \theta)>0$ is sufficiently large, then

$$
V\left(\mathcal{P}^{-1}(\zeta, \eta) ; \theta\right)-V(\zeta, \eta ; \theta) \leq-\varepsilon .
$$

Thus, there are disjoint half-planes $V(\zeta, \eta ; \theta) \geq c$ and $V(\zeta, \eta ; \theta) \leq-c$ such that any sequence

$$
\{\zeta, \eta\}, \mathcal{P}(\zeta, \eta), \ldots, \mathcal{P}^{k}(\zeta, \eta), \ldots
$$

starting in the first half-plane and any sequence

$$
\{\zeta, \eta\}, \mathcal{P}^{-1}(\zeta, \eta), \ldots, \mathcal{P}^{-k}(\zeta, \eta), \ldots
$$

starting in the second half-plane belong to the same half-planes as their initial values and go to infinity. Therefore the solution $x(t ; \zeta, \eta)$ of equation (9) satisfies the first of estimates (16) whenever $\{\zeta, \eta\}$ belongs to the half-plane $V(\zeta, \eta ; \theta) \geq c$ and the second of estimates (16) whenever $\{\zeta, \eta\}$ belongs to the half-plane $V(\zeta, \eta ; \theta) \leq-c$.

Let us choose another $\theta$ such that (47) holds. For this $\theta$ we can repeat all the arguments exactly in the same way as above. Consequently, there is another pair of half-planes consisting of the initial values of unbounded solutions. The unity of these four half-planes is the whole plane $\{\zeta, \eta\}$ without a bounded set (a parallelogram). Any solution with the initial value out of this parallelogram satisfies at least one of relations (16).

Theorem 6 is proved. The main idea of the proof for bounded $f$ was published in $[1]$.

\section{Proof of Theorem 7}

The proof of this theorem is close to that of the main result from [12], some ideas are similar to the proof of Theorem 4 from that paper. The computations of the index at infinity of the vector field $\Phi x=x-\mu A x-A F(x)$ are different for different conclusions of Theorem 7 . Here and below by $F(x)$ we denote the superposition operator $F(x(\cdot))=$ $f(\cdot, x(\cdot))$.

11.1 Conclusion (i). Let $\varphi^{+}<0<\varphi_{-}$(the case $\varphi^{-}<0<\varphi_{+}$can be studied in a similar way). Set $p(x)=(g, x)_{L^{2}}$ and $Q x=x-p(x) e$ for all $x \in L^{2}=L^{2}(a, b)$ and consider the deformation

$$
\Xi_{1}(\lambda, x)=Q x-\mu A Q x-\lambda A F(x)+(1-\lambda) \mu^{-1} s(p(x)) e \quad(\lambda \in[0,1])
$$


where $s$ is function (39). Since $e=\mu A e$, the equalities

$$
\begin{aligned}
& \Xi_{1}(0, x)=Q x-\mu A Q x+\mu^{-1} s(p(x)) e \\
& \Xi_{1}(1, x)=\Phi(x)
\end{aligned}
$$

are valid. To prove conclusion (i) of Theorem 7 it suffices to establish the relation $\left|\operatorname{ind}_{\infty} \Xi_{1}(0, \cdot)\right|=1$ and to prove an a priori estimate $\|x\|_{L^{2}} \leq$ const $<\infty$ for all the zeroes of the deformation $\Xi_{1}$.

The first assertion is simple, since the equation $\Xi_{1}(0, x)=0$ has a unique solution $x=0$ and the index of this solution equals either 1 or -1 . This follows from the rotation product formula cited above. Indeed, $\Xi_{1}(0, \cdot)$ is the direct sum of the scalar field $\chi(\xi e)=\mu^{-1} s(\xi) e \quad(\xi \in \mathbb{R})$ and the vector field $h-\mu A h \in E$, where $E=Q L^{2}$ is a subspace of $L^{2}$ with co-dimension 1 . The index of the zero $\xi=0$ of the scalar field $\chi$ equals $\operatorname{sgn} \mu$, while the index of the zero $h=0$ of the vector field $h-\mu A h \in E$ equals $(-1)^{\beta}$ where $\beta$ is the sum of the multiplicities of all the real eigenvalues of the operator $\mu A$ that are greater than 1 , hence $\operatorname{ind}_{\infty} \Xi_{1}(0, \cdot)=(-1)^{\beta} \operatorname{sgn} \mu$.

Now let us prove an a priori estimate for the zeroes of $\Xi_{1}$. If $\Xi_{1}(\lambda, x)=0$ for some $x=\xi e+h \quad(h \in E)$ and $\lambda \in[0,1]$, then

$$
\begin{aligned}
& 0=Q \Xi_{1}(\lambda, x)=h-\mu A h-\lambda A Q F(\xi e+h) \\
& 0=\mu p\left(\Xi_{1}(\lambda, x)\right)=-\lambda p(F(\xi e+h))+(1-\lambda) s(\xi) .
\end{aligned}
$$

The first equality rewritten as $h=\lambda(I-\mu A Q)^{-1} A Q F(x)$ implies the estimate $\|h\|_{C^{1}} \leq$ $c \Theta(|\xi|)$. Consider the second equality. If $|\xi| \geq 1$, then $s(\xi)=\operatorname{sgn} \xi$. Theorems 1 and 2 imply

$$
p(F(\xi e+h))-p(F(\xi e))=p(F(\xi e+h))-\Psi(\xi) \rightarrow 0 \quad(\xi \rightarrow+\infty),
$$

hence

$$
\mu p\left(\Xi_{1}(\lambda, x)\right)+\lambda \Psi(\xi)-(1-\lambda) \operatorname{sgn} \xi \rightarrow 0 \quad(\xi \rightarrow \pm \infty)
$$

and therefore

$$
\mu p\left(\Xi_{1}(\lambda, x)\right)\left\{\begin{array}{l}
\geq 1-\lambda-\lambda \varphi^{+}+o(1) \quad \text { as } \xi \rightarrow+\infty \\
\leq \lambda-1-\lambda \varphi_{-}+o(1) \quad \text { as } \xi \rightarrow-\infty
\end{array}\right.
$$

Since

$$
\begin{aligned}
& 1-\lambda-\lambda \varphi^{+} \geq \min \left\{1,-\varphi^{+}\right\}>0 \\
& \lambda-1-\lambda \varphi_{-} \leq-\min \left\{1, \varphi_{-}\right\}<0
\end{aligned}
$$

we see that system (48) - (49) has no solutions if $|\xi|$ is sufficiently large. This proves the required a priori estimate.

11.2 Conclusion (ii). To be definite, consider the case $\varphi_{-}>0$ and $\varphi_{+}>0$ (the other case $\varphi^{-}<0$ and $\varphi^{+}<0$ can be studied is similarly). Define the deformation

$$
\Xi_{2}(\lambda, x)=Q x-\mu A Q x-\lambda A F(x)-(1-\lambda) \mu^{-1} \varphi_{+} e \quad(\lambda \in[0,1]) .
$$


Now $\Xi_{2}(1, x)=\Phi(x)$ and $\Xi_{2}(0, x)=Q x-\mu A Q x-\mu^{-1} \varphi_{+} e$; the equation $\Xi_{2}(0, x)=0$ has no solutions and therefore $\operatorname{ind}_{\infty} \Xi_{2}(0, \cdot)=0$. To prove the conclusion (ii) of Theorem 7 , it suffices to establish an a priori estimate $\|x\|_{L^{2}} \leq$ const $<\infty$ for all the zeroes $x=\xi e+h$ of the deformation $\Xi_{2}$. This a priori estimate follows from the estimate $\|h\|_{C^{1}} \leq c \Theta(|\xi|)$ and the relations

$$
\mu p\left(\Xi_{2}(\lambda, x)\right)+\lambda \Psi(\xi)+(1-\lambda) \varphi_{+}=-\lambda p(F(x))+\lambda p(F(\xi e)) \rightarrow 0 \quad(\xi \rightarrow \pm \infty)
$$

and

$$
\liminf _{\xi \rightarrow \pm \infty}\left(\lambda \Psi(\xi)+(1-\lambda) \varphi_{+}\right) \geq \min \left\{\varphi_{+}, \varphi_{-}\right\}>0 .
$$

11.3 Conclusion (iii). Let $\varphi_{+}<0<\varphi^{+}$(we omit the similar proof for the case $\left.\varphi_{-}<0<\varphi^{-}\right)$. We need to prove the existence of zeroes $x_{n}=\xi_{n} e+h_{n}$ of the vector field $\Phi$ with norms $\left\|x_{n}\right\|_{L^{2}} \rightarrow \infty$. As everywhere above, for each zero $x=\xi e+h$ the equality $Q \Phi(x)=0$ implies estimates (42). Set $\varepsilon=\frac{1}{3} \min \left\{\varphi^{+},-\varphi_{+}\right\}$and fix a $\xi_{0}$ such that

$$
|p(F(\xi e+h))-\Psi(\xi)|<\varepsilon
$$

for all $\xi \geq \xi_{0}$ and $\|h\|_{C^{1}} \leq c \Theta(\xi)$ where $c>0$ comes from (42); the existence of such $\xi_{0}$ follows from (4). By definition of $\varphi_{+}$and $\varphi^{+}$, there exist numbers $\xi_{*}>\xi_{0}$ and $\xi^{*}>\xi_{*}$ such that

$$
\Psi\left(\xi_{*}\right)<-2 \varepsilon \quad \text { and } \quad \Psi\left(\xi^{*}\right)>2 \varepsilon .
$$

Consider the set

$$
\Omega=\left\{x \in L^{2}:\|Q x\|_{L^{2}} \leq R_{1} \Theta\left(\xi^{*}\right)+1, p(x) \in\left[\xi_{*}, \xi^{*}\right]\right\}
$$

where $R_{1}$ is defined in (42). The boundary $\partial \Omega$ of $\Omega$ consists of the three parts

$$
\begin{aligned}
G_{*} & =\left\{\|Q x\|_{L^{2}} \leq R_{1} \Theta\left(\xi^{*}\right)+1, p(x)=\xi_{*}\right\} \\
G^{*} & =\left\{\|Q x\|_{L^{2}} \leq R_{1} \Theta\left(\xi^{*}\right)+1, p(x)=\xi^{*}\right\} \\
G & =\left\{\|Q x\|_{L^{2}}=R_{1} \Theta\left(\xi^{*}\right)+1, p(x) \in\left[\xi_{*}, \xi^{*}\right]\right\} .
\end{aligned}
$$

The vector field $\Phi$ is non-zero on $G$ due to the estimates $\|h\|_{L^{2}} \leq R_{1} \Theta(\xi) \leq R_{1} \Theta\left(\xi^{*}\right)$ which are valid for all the solutions $x=\xi e+h$ of $Q \Phi(x)=0$ in $\Omega$. Relations (50) - (51) imply

$$
\begin{array}{lll}
\mu p(\Phi(x))=-p(F(x))+\Psi(p(x))-\Psi(p(x))>-\varepsilon+2 \varepsilon=\varepsilon>0 & & \left(x \in G_{*}\right) \\
\mu p(\Phi(x))=-p(F(x))+\Psi(p(x))-\Psi(p(x))<\varepsilon-2 \varepsilon=-\varepsilon<0 & & \left(x \in G^{*}\right)
\end{array}
$$

and $\|h\|_{C^{1}} \leq c \Theta(\xi)$, hence $\Phi(x) \neq 0$ on $G_{*} \cup G^{*}$. Thus $\Phi \neq 0$ on $\partial \Omega$. We also see that $\mu p(\Phi(x))>0$ if $Q \Phi(x)=0, p(x)=\xi_{*}$ and $\mu p(\Phi(x))<0$ if $Q \Phi(x)=0, p(x)=\xi^{*}$.

Now we can calculate the rotation $\gamma(\Phi, \partial \Omega)$ of the field $\Phi$ on $\partial \Omega$ using an appropriate deformation and [10: Theorem 3]. Let us reformulate this theorem for our problem.

Let $X$ be a Banach space, let some completely continuous operator $\mathcal{A}=\left\{A_{1}, A_{2}\right\}$ be defined on $\Omega=\Omega_{1} \times \Omega_{2}$ where $\Omega_{1}$ is the ball $\|x\|_{X} \leq r$ and $\Omega_{2}=\left[\xi_{*}, \xi^{*}\right]$, let for any $\xi \in \Omega_{2}$ the vector field $x-A_{1}(x, \xi)$ be non-degenerate on $\partial \Omega_{1}=\left\{\|x\|_{X}=r\right\}$ and let the rotation $\gamma_{1}=\gamma\left(I-A_{1}(\cdot, \xi), \partial \Omega_{1}\right)$ be non-zero. Then $\gamma_{1}$ is the same for all $\xi \in \Omega_{2}$. Denote by $K(\xi)$ the non-empty set of solutions $x$ of the equation $x=A_{1}(x, \xi)$ for each $\xi \in \Omega_{2}$. 
Statement 1. If $\xi_{*}-A_{2}\left(x, \xi_{*}\right)<0$ for all $x \in K\left(\xi_{*}\right)$ and $\xi^{*}-A_{2}\left(x, \xi^{*}\right)>0$ for all $x \in K\left(\xi^{*}\right)$, then $\gamma(\mathcal{A}, \partial \Omega)=\gamma_{1}$.

In our case, this statement implies that either $\gamma(\Phi, \partial \Omega)=1$ or $\gamma(\Phi, \partial \Omega)=-1$. Therefore there exists at least one zero $x_{*} \subset \Omega$ of the vector field $\Phi$; relation $x_{*} \in \Omega$ implies $\left(g, x_{*}\right)_{L^{2}} \geq \xi_{*}$. Since the numbers $\xi_{*}$ and $\xi^{*}$ satisfying (51) may be chosen arbitrarily large, there is a sequence $\left\{x_{n}\right\}$ of zeroes of the vector field $\Phi$ with unbounded norms: $\left\|x_{n}\right\|_{L^{2}} \rightarrow \infty$. Theorem 7 is proved.

\section{Proof of Theorem 9}

12.1 The choice of unknowns. First, let us rescale the time in (22). For any $w \in \Omega=\left[w_{1}, w_{2}\right]$ every $2 \pi$-periodic solution $x(t)$ of the equation

$$
L\left(w \frac{d}{d t}\right) x=f(x)
$$

determines the $\frac{2 \pi}{w}$-periodic solution $x(w t)$ of equation (22). We consider (52) instead of (22) and prove that for some $w \in \Omega$ equation (52) has at least one non-trivial $2 \pi$ periodic solution $x(t)=r \sin t+h(t)$ where $r>0, h$ is $2 \pi$-periodic and $P h=0$, with $P$ defined by

$$
(P x)(t)=\frac{1}{\pi} \int_{0}^{2 \pi} \cos (t-\tau) x(\tau) d \tau .
$$

Let us stress that any non-stationary periodic solution $x$ of autonomous equation (52) generates the continuum of periodic solutions $x(t+\alpha)$. We delete the non-uniqueness by fixing the solution that has zero projection onto cos $t$ and a positive projection onto $\sin t$. At the same time, the frequency $w$ is considered as an additional unknown.

12.2 Linear subspaces and operators. The first linear operator is the projector $P$. Set $Q=I-P$ and define the subspaces $\Pi=P L^{2}$ and $\Pi^{*}=Q L^{2}$ in $L^{2}=L^{2}(0,2 \pi)$. These subspaces are orthogonal, $\Pi$ is two-dimensional and $\Pi^{*}$ has co-dimension 2 .

Denote by $A(w) \quad(w \in \Omega)$ the linear operator that maps any function $u \in \Pi^{*}$ to a unique solution $x \in \Pi^{*}$ of the linear equation

$$
L\left(w \frac{d}{d t}\right) x=u(t)
$$

Consider the operators $A(w) Q$. Their norms in $L^{2}$ are uniformly bounded:

$$
\|A(w) Q\|_{L^{2} \rightarrow L^{2}}=q_{*}(w) \leq c_{*}:=\sup _{w \in \Omega} q_{*}(w)<\infty, \quad q_{*}(w)=\sup _{ \pm 1 \neq n \in \mathbb{Z}}|L(n w i)|^{-1} .
$$

Each operator $A(w) Q$ is completely continuous from $L^{2}$ to $C$ and continuous from $C$ to $C^{1}$. Moreover, $A(\cdot) Q: \Omega \times L^{2} \rightarrow L^{2}$ is completely continuous with respect to both arguments $w \in \Omega$ and $u \in L^{2}$.

We use the notation $P_{n}$ for the following orthogonal projectors in $L^{2}$. By $P_{0}$ we denote the orthogonal projector onto the one-dimensional subspace $\Pi_{0}$ of constant functions; $P_{n}$ with $n \geq 2$ is the projector onto the two-dimensional linear span $\Pi_{n}$ of the functions $\sin n t$ and $\cos n t$. Each subspace $\Pi_{n}$ is invariant for the operator $A(w) Q$. The one-dimensional subspace $\Pi_{0}$ corresponds to the eigenvalue $(L(0))^{-1}$; each plane $\Pi_{n}$ corresponds to the pair $(L( \pm n w i))^{-1}$. 
Lemma 3. Let $w \in \Omega$. The functions $x=r \sin +h \quad\left(h \in \Pi^{*}\right)$ and $u \in L^{2}$ satisfy equation (53) if and only if $h=A(w) Q u$ and

$$
\begin{aligned}
& \pi r \operatorname{Re} L(w i)=\int_{0}^{2 \pi} \sin t u(t) d t \\
& \pi r \operatorname{Im} L(w i)=\int_{0}^{2 \pi} \cos t u(t) d t .
\end{aligned}
$$

Relations $h=A(w) Q u \quad(u \in C)$ imply

$$
\left(P_{n} h^{\prime}, P_{n} u\right)_{L^{2}}=n \operatorname{Im} L(n w i)\left\|P_{n} h\right\|_{L^{2}}^{2} \quad(n \geq 2) .
$$

The proof is by simple computations and we omit it.

12.3 Main deformation. By Lemma 3 , the problem on $2 \pi$-periodic solutions of equation (52) is equivalent to the system

$$
\left.\begin{array}{rl}
\pi \operatorname{Re} L(w i) & =\frac{1}{r} \int_{0}^{2 \pi} \sin t f(r \sin t+h(t)) d t \\
\pi \operatorname{Im} L(w i) & =\frac{1}{r} \int_{0}^{2 \pi} \cos t f(r \sin t+h(t)) d t \\
h & =A(w) Q f(r \sin t+h(t))
\end{array}\right\} .
$$

Consider in the space $\mathbb{E}=\mathbb{R} \times \mathbb{R} \times \Pi^{*}$ the deformation

$$
\Phi_{\lambda}(r, w, h)=\left\{\begin{array}{l}
\lambda \pi \operatorname{Re} L(w i)-\frac{1}{r} \int_{0}^{2 \pi} \sin t f(r \sin t+\lambda h(t)) d t \\
\pi \operatorname{Im} L(w i)-\frac{\lambda}{r} \int_{0}^{2 \pi} \cos t f(r \sin t+h(t)) d t \\
h-\lambda A(w) Q f(r \sin t+h(t))
\end{array}\right.
$$

of the vector field $\Phi_{1}$ to the field

$$
\Phi_{0}(r, w, h)=\left\{\begin{array}{l}
-\frac{1}{r} \int_{0}^{2 \pi} \sin t f(r \sin t) d t \\
\pi \operatorname{Im} L(w i) \\
h .
\end{array}\right.
$$

Here $\lambda \in[0,1]$ is the parameter of deformation. For $\lambda=1$ any zero of the vector field $\Phi_{1}$ is a solution of system (54); we should prove the existence of such zeroes. For $\lambda=0$ the vector field $\Phi_{0}$ has a simple form and it is easy to compute its topological characteristics on proper sets.

12.4 Rotation of the vector field $\Phi_{0}$. Consider the set

$$
G=G\left(\rho, R, w_{1}, w_{2}, c_{1}\right):=\left\{r \in[\rho, R], w \in\left[w_{1}, w_{2}\right],\|h\|_{L^{2}} \leq c_{1} \Theta(R)\right\} \subset \mathbb{E} .
$$

The positive constants $\rho, R, c_{1}$ are determined by the values $w_{1}, w_{2}, q$, the polynomial $L$ and the function $f$. The number $\rho$ is sufficiently small, the numbers $R$ and $c_{1}$ are sufficiently large. If we choose the parameters $\rho, R$, and $c_{1}$ such that deformation (55) 
is non-degenerate on the boundary $\partial G$ of the domain $G$ and if the rotation $\gamma\left(\Phi_{0}, \partial G\right)$ of the vector field $\Phi_{0}$ on $\partial G$ is non-zero, then Theorem 9 will be proved.

By the rotation product formula, $\gamma\left(\Phi_{0}, \partial G\right)$ is the product of three rotations. The first is the rotation $\gamma_{r}$ of the scalar vector field

$$
-\frac{1}{r} \int_{0}^{2 \pi} \sin t f(r \sin t) d t=-\frac{\Psi(r)}{r}
$$

on the boundary of the interval $[\rho, R]$; the second is the rotation $\gamma_{w}$ of the scalar field $\pi \operatorname{Im} L(w i)$ on the boundary of the interval $\Omega$; the third is the rotation $\gamma_{h}$ of the vector field $h$ on the sphere $\left\{h \in \Pi^{*}:\|h\|_{L^{2}}=c_{1} \Theta(R)\right\}$.

The rotation $\gamma_{r}$ is either 1 or -1 . This follows from condition (25): for small $r=\rho$ component (56) of $\Phi_{0}$ has the same sign as $-f^{\prime}(0)$; for large $r=R$ this component has the opposite sign coinciding with the sign of $-\Psi(R)$. Of course, $\rho$ should be sufficiently small and $R$ should be sufficiently large.

The rotation $\gamma_{w}$ is also either 1 or -1 . The component $\pi \operatorname{Im} L(w i)$ has opposite signs for $w=w_{1}$ and $w=w_{2}$, since the polynomial $\operatorname{Im} L(w i)$ has a unique root $w_{0}$ inside $\left[w_{1}, w_{2}\right]$ and this root is of odd multiplicity.

The rotation $\gamma_{h}$ equals 1 . We see that $\gamma\left(\Phi_{0}, \partial G\right) \neq 0$.

12.5 Auxiliary estimates. Below we use the notation $\|\cdot\|=\|\cdot\|_{L^{2}}$.

Lemma 4. Let $w \in \Omega, q_{1}(w):=|\operatorname{Im} L(w i)|$ and $q_{2}(w):=\inf _{n \in \mathbb{N}_{0} \backslash\{1\}}|L(n w i)|$. If the second and the third components of deformation (55) are zero, then $q_{1}(w) \leq q$ and

$$
\|h\| \leq r \sqrt{\pi \frac{q^{2}-q_{1}^{2}(w)}{q_{2}^{2}(w)-q^{2}}} .
$$

Proof. We have the implications

$$
\begin{aligned}
h=\lambda A(w) Q f(r \sin t+h) & \Longrightarrow \\
\pi r \operatorname{Im} L(w i)=\lambda \int_{0}^{2 \pi} \cos t f(x(t)) d t & \Longrightarrow \sqrt{\pi} r|\operatorname{Im} L(w i)| \lambda\|P f(x)\|
\end{aligned}
$$

for $n \in \mathbb{N}_{0} \backslash\{1\}$. Therefore

$$
\pi r^{2}|\operatorname{Im} L(w i)|^{2}+\sum_{n \in \mathbb{N}_{0} \backslash\{1\}}|L(n w i)|^{2}\left\|P_{n} h\right\|^{2} \leq \lambda^{2}\|f(x)\|^{2}
$$

and from $\lambda \leq 1$ it follows that

$$
\begin{aligned}
& \pi r^{2}|\operatorname{Im} L(w i)|^{2}+\sum_{n \in \mathbb{N}_{0} \backslash\{1\}}|L(n w i)|^{2}\left\|P_{n} h\right\|^{2} \\
& \quad \leq q^{2}\|x\|^{2} \\
& \quad=q^{2}\left(\pi r^{2}+\sum_{n \in \mathbb{N}_{0} \backslash\{1\}}\left\|P_{n} h\right\|^{2}\right),
\end{aligned}
$$

consequently

$$
\pi r^{2} q_{1}^{2}(w)+q_{2}^{2}(w)\|h\|^{2} \leq q^{2}\left(\pi r^{2}+\|h\|^{2}\right)
$$

where $q<q_{2}(w)$ by assumption. Hence the conclusion of Lemma 4 is true 
Lemma 5. There exist numbers $c, c_{2}>0$ such that $\|h\|_{L^{2}},\|h\|_{C^{1}} \leq$ cr and $\|h\|_{L^{2}},\|h\|_{C^{1}} \leq \mathbb{\square}$ $c_{2} \Theta(r)$ for any zero $\{r, w, h\}$ of deformation (55).

This lemma follows from Lemma 4 and from the properties of the operators $A(w) Q$.

12.6 Non-degeneracy of $\boldsymbol{\Phi}_{\boldsymbol{\lambda}}$. To complete the proof of Theorem 9 , it remains to show that deformation (55) is non-zero on the boundary $\partial G$ of the domain $G$ for some sufficiently small $\rho$ and sufficiently large $R$ and $c_{1}$. The boundary $\partial G$ is the join of the sets

$$
\begin{aligned}
G_{\rho} & =\left\{r=\rho, w \in\left[w_{1}, w_{2}\right],\|h\| \leq c_{1} \Theta(R)\right\} \\
G_{R} & =\left\{r=R, w \in\left[w_{1}, w_{2}\right],\|h\| \leq c_{1} \Theta(R)\right\} \\
G_{w} & =\left\{r \in[\rho, R], w=w_{j}(j=1,2),\|h\| \leq c_{1} \Theta(R)\right\} \\
G_{h} & =\left\{r \in[\rho, R], w \in\left[w_{1}, w_{2}\right],\|h\|=c_{1} \Theta(R)\right\} .
\end{aligned}
$$

Let $\Phi_{\lambda}(r, w, h)=0$ for some $\{r, w, h\} \in G$ and $\lambda \in[0,1]$. Then

$$
\lambda^{2} \frac{\operatorname{Re} L(w i)}{\operatorname{Im} L(w i)} \int_{0}^{2 \pi} \cos t f(r \sin t+h(t)) d t=\int_{0}^{2 \pi} \sin t f(r \sin t+\lambda h(t)) d t .
$$

First consider this equality for small $r$. By assumption, $w_{0}$ is a root of the same multiplicity $K$ for the polynomials $L(i w)$ and $\operatorname{Im} L(i w)$. This means that the multiplicity of this root for the polynomial $\operatorname{Re} L(i w)$ is greater or equal than $K$, therefore the function $\frac{\operatorname{Re} L(w i)}{\operatorname{Im} L(w i)}$ is continuous on $\Omega$. Since $f(x)=f^{\prime}(0) x+o(x) \quad(x \rightarrow 0)$, the term of order $r$ in the left-hand side of (58) equals zero. In the right-hand side, the term of order $r$ equals

$$
\int_{0}^{2 \pi} \sin t f^{\prime}(0)(r \sin t+\lambda h(t)) d t=\pi f^{\prime}(0) r
$$

i.e. this term is non-zero. The other terms have smaller order with respect to $r$ due to Lemma 5. Therefore equality (58) cannot be valid for sufficiently small $r$, hence $\{r, w, h\} \notin G_{\rho}$ if $\rho$ is sufficiently small.

The relation $\{r, w, h\} \notin G_{R}$ for sufficiently large $R$ follows from (58) due to Theorems 1 and 2. These theorems imply

$$
\begin{aligned}
\int_{0}^{2 \pi} \cos t f(r \sin t+h(t)) d t & \rightarrow 0 \\
\int_{0}^{2 \pi} \sin t f(r \sin t+\lambda h(t)) d t-\int_{0}^{2 \pi} \sin t f(r \sin t) d t & \rightarrow 0
\end{aligned}
$$

and from (25) it follows that

$$
\left|\int_{0}^{2 \pi} \sin t f(r \sin t+\lambda h(t)) d t\right| \geq \frac{c_{0}}{2\left|f^{\prime}(0)\right|}>0
$$

for all $r \geq r_{0}>0$. Therefore relation (58) cannot be valid for large $r$. 
The proof of relation $\{r, w, h\} \notin G_{w}$ is the most cumbersome. Since

$$
\int_{0}^{2 \pi} f(r \sin t+h(t))\left(r \cos t+h^{\prime}(t)\right) d t=0
$$

and the second component of (55) is zero, we have

$$
\pi \operatorname{Im} L(w i) r^{2}+\lambda \int_{0}^{2 \pi} h^{\prime}(t) f(r \sin t+h(t)) d t=0 .
$$

Equivalently,

$$
\pi \operatorname{Im} L(w i) r^{2}+\lambda \sum_{n \geq 2} \int_{0}^{2 \pi} P_{n} h^{\prime}(t) P_{n} f(r \sin t+h(t)) d t=0 .
$$

But $h(t)=\lambda A(w) Q f(r \sin t+h(t))$ and, by Lemma 3,

$$
\lambda \int_{0}^{2 \pi} P_{n} h^{\prime}(t) P_{n} f(r \sin t+h(t)) d t=n \operatorname{Im} L(w n i)\left\|P_{n} h\right\|^{2} \quad(n \geq 2),
$$

therefore

$$
\pi \operatorname{Im} L(w i) r^{2}+\sum_{n \geq 2} n \operatorname{Im} L(w n i)\left\|P_{n} h\right\|^{2}=0,
$$

i.e.

$$
\pi|\operatorname{Im} L(w i)| r^{2}+\operatorname{sgn}(\operatorname{Im} L(w i)) \sum_{n \geq 2} n \operatorname{Im} L(w n i)\left\|P_{n} h\right\|^{2}=0 .
$$

From relation (57) rewritten as

$$
\sum_{n \in \mathbb{N}_{0} \backslash\{1\}}\left(|L(w n i)|^{2}-q^{2}\right)\left\|P_{n} h\right\|^{2} \leq \pi r^{2}\left(q^{2}-|\operatorname{Im} L(w i)|^{2}\right)
$$

the estimate

$$
\begin{aligned}
& \sum_{n \in \mathbb{N}_{0} \backslash\{1\}}\left(|\operatorname{Im} L(w i)|\left(|L(n w i)|^{2}-q^{2}\right)\right. \\
& \left.\quad+n\left(q^{2}-|\operatorname{Im} L(w i)|^{2}\right) \operatorname{sgn}(\operatorname{Im} L(w i)) \operatorname{Im} L(n w i)\right)\left\|P_{n} h\right\|^{2} \leq 0
\end{aligned}
$$

follows. Due to (23), this estimate is impossible for $w=w_{j} \quad(j=1,2)$ if $h \neq 0$. If $h=0$, then the second component of (55) equals $\pi \operatorname{Im} L(w i)$ and differs from zero for $w=w_{j} \quad(j=1,2)$. Therefore in both cases $\{r, w, h\} \notin G_{w}$.

Finally, Lemma 5 implies $\{r, w, h\} \notin G_{h}$ if we choose $c_{1}>c_{2}$. Theorem 9 is completely proved. 


\section{References}

[1] Alonso, J. M. and R. Ortega: Unbounded solutions of semilinear equations at resonance. Nonlinearity 9 (1996), 1099 - 1111.

[2] Bliman, P.-A., Krasnosel'skii, A. M. and D. I. Rachinskii: Sector estimates of nonlinearities and self-oscillation existence in control systems. Automat. Remote Control 61 (2000), $889-903$.

[3] Bobylev, N. A., Burman, M. Yu. and S. K. Korovin: Approximation Procedures in Nonlinear Oscillation Theory. Berlin - New York: W. de Gruyter 1994.

[4] Dancer, E. N.: On the use of asymptotics in nonlinear boundary value problems. Ann. Mat. Pure Appl. 4 (1982), 167 - 185.

[5] Gaines, R. E. and J. Mawhin: Coincidence Degree and Nonlinear Differential Equations. Lect. Notes Math. 568 (1977).

[6] Krasnosel'skii, A. M.: Asymptotic homogeneity of hysteresis operators. Z. Angew. Math. Mech. (ZAMM) 76 (1996), 313 - 316.

[7] Krasnosel'skii, A. M.: On forced oscillations in systems with even functional characteristics. Automat. Remote Control 58 (1997), 27 - 36.

[8] Krasnosel'skii, A. M.: On oscillations in resonant equations with complex nonlinearities. In: Proc. 7th IEEE Mediterranean Conf. on Control and Automation. Haifa (Israel) 1999, pp. $1231-1243$.

[9] Krasnosel'skii, A. M. and M. A. Krasnosel'skii: Large-amplitude cycles in autonomous systems with hysteresis. Soviet Math. Dokl. 32 (1985), $14-17$.

[10] Krasnosel'skii, A. M. and M. A. Krasnosel'skii: Vector fields in the product of spaces and applications to differential equations. Diff. Equ. 33 (1997), 59 -66.

[11] Krasnosel'skii, A. M. and J. Mawhin: Periodic solutions of equations with oscillating nonlinearities. Math. Comput. Modelling 32 (2000), 1445 - 1455.

[12] Krasnosel'skii, A. M. and J. Mawhin: The index at infinity for some vector fields with oscillating nonlinearities. Discrete Contin. Dyn. Systems 6 (2000), $165-174$.

[13] Krasnosel'skii, A. M. and D. I. Rachinskii: Hopf bifurcations from infinity, generated by bounded nonlinear terms. Funct. Differ. Equ. 6 (1999), 357 - 374.

[14] Krasnosel'skii, A. M. and D. I. Rachinskii: On Hamiltonian nature of Lurie systems. Automat. Remote Control 61 (2000), 1259 - 1262.

[15] Krasnosel'skii, A. M. and D. I. Rachinskii: Existence of cycle continua in Hamiltonian control systems. Automat. Remote Control 62 (2001), 227 - 235.

[16] Krasnosel'skiı̌, M. A. and P. P. Zabreйiko: Geometrical Methods of Nonlinear Analysis. Berlin: Springer-Verlag 1984.

[17] Lazer, A. C. and D. E. Leach: Bounded perturbations of forced harmonic oscillators at resonance. Ann. Mat. Pure Appl. 82 (1969), 46 - 68.

[18] Mawhin, J. and M. Willem: Critical Point Theory and Hamiltonian Systems (Appl. Math. Sci.: Vol. 74). New York et al.: Springer-Verlag 1989.

Received 12.09.2001 